\title{
Single-variable porous nanomaterial series from polymer structure-directing agents
}

\author{
Morgan Stefiik ${ }^{1,2, a)}$ (1) \\ ${ }^{1}$ Department of Chemistry and Biochemistry, University of South Carolina, Columbia, SC 29208, USA \\ ${ }^{2}$ Director of South Carolina SAXS Collaborative, University of South Carolina, Columbia, SC 29208, USA \\ a) Address all correspondence to this author. e-mail: morgan@stefikgroup.com \\ Received: 1 June 2021; accepted: 21 October 2021; published online: 2 December 2021
}

Block polymer structure-directing agents (SDA) enable the production of porous nanoscale materials. Most strategies rely upon polymer equilibration where diverse morphologies are realized in porous functional materials. This review details how solvent selectivity determines the polymer SDA behaviors, spanning from bulk-type to solution-type. Equilibrating behavior of either type, however, obscures nanostructure cause-and-effect since the resulting sample series convolve multiple spatial variations. Solution-type SDA behaviors include both dynamic and persistent micelles. Persistent micelle templates (PMT) use high solvent selectivity for kinetic entrapment. PMTs enable independent wall thickness control with demonstrated $2 \AA$ precision alterations. Unimodal PMT pore size distributions have spanned from 11.8 to $109 \mathrm{~nm}$ and multimodal pore sizes up to $290 \mathrm{~nm}$. The PMT method is simple to validate with diffraction models and is feasible in any laboratory. Finally, recent energy device publications enabled by PMT are reviewed where tailored nanomaterials provide a unique perspective to unambiguously identify nanostructure-property-performance relationships.

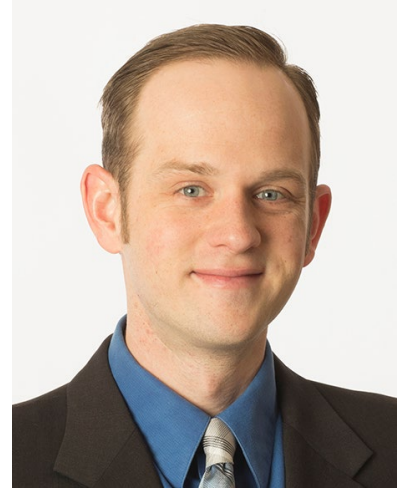

Morgan Stefik is an Associate Professor in the Department of Chemistry and Biochemistry at the University of South Carolina and is the founding Director of the South Carolina SAXS Collaborative. He obtained his BE degree in Materials Engineering from California Polytechnic State University in 2005, and his $\mathrm{PhD}$ degree in Materials Science from Cornell University in 2010. He then completed post-doctoral research at École Polytechnique Fédérale de Lausanne. His awards include a National Science Foundation CAREER Award in 2018, recognition as an Emerging Investigator by the Journal of Materials Chemistry A in 2017, a Breakthrough Star Award from the University of South Carolina in 2018, election to the council of the International Mesostructured Materials Association in 2018, and selection as an American Chemical Society Division of Polymeric Materials: Science and Engineering division Young Investigator in 2020. Stefik's interests include nanomaterial synthesis, characterization, and fundamental energy device studies. Many of the research projects have a theme of seeking simplicity within a context of complexity. On the synthesis side, the group focuses on precision nanomaterials chemistry with techniques that enable atomic level control such as PMT or atomic layer deposition. Polymer physics plays a large role in many projects where control over the molecular-scale behaviors of polymers translates into greater control over the produced porous materials. On the device side the group has advanced solar water splitting devices and energy storage materials. 


\section{Introduction and background}

The materials science tetrahedron is introduced in undergraduate classes to connect the foundational relationships between processing, structure, properties, and performance. The tetrahedron is often introduced from the perspective of atom-based crystals (e.g., metals and ceramics), however, this framework applies equally well for nanomaterials science. The processing of nanomaterials determines their nanostructure (architecture) which in turn determines the properties and performance. For porous materials, the complete nanoscale architecture including both the material and the porosity influence performance. Over the past decades, numerous nanomaterial synthesis approaches were developed $[1,2]$, each with some benefits and drawbacks. For example, methods that directly produce nanoparticles such as hydrothermal [3] or precipitation [4] reactions have control over the material dimensions, shape, and crystallography but require additional processing to yield porosity. Similarly, deposition methods such as physical or chemical vapor deposition enable a variety of nanostructured motifs such as columnar, branched, and granular morphologies [5-7], however, the architectural dimensions often vary with deposition thickness. Other methods based on hard templates such as opal templates [8] enable diverse material compositions and high-temperature annealing/crystallization prior to template removal, however, the resulting porosity is a function of both the template and the fraction of material infilling. Similarly, anodic etching methods [9] yield diverse porous nanomaterials, however, the processing-nanostructure correlations are complex. Yet, other methods based on metal organic frameworks $[10,11]$ have modular control over porosity and chemical functionality, however, the pore sizes are typically less than a few $\mathrm{nm}$ in diameter. Among these and other nanomaterial synthesis methods, block polymer structure-directing agents (SDA) stand out in terms of simple solution processing that defines both the porosity and the material dimension simultaneously while spanning device-relevant length scales from a few nanometers to hundreds of nanometers. The study of nanostructure-property relationships is most simple when a single architectural attribute is altered at a time. Towards this end, a processing route called persistent micelle templates (PMT) was recently developed [12] which enables tailored variations of porous nanomaterial series with one architectural variable altered at a time in a predictable and simple manner. This review article summarizes numerous block polymer SDA behaviors to place recent PMT developments within a broader context. Also recent PMT-enabled studies are discussed where new nanostructure-property-performance relationships were revealed.

The natural self-assembly of block polymers into wellordered morphologies has inspired many to seek the same spatial control in the form of diverse functional materials [13]. One of the most convenient paths towards this end is to use block polymers as SDAs that organize material precursors from solutions either via evaporation or precipitation $[14,15]$. Here, a selective interaction, often hydrogen bonding or a coulombic interaction, is generally used to associate the material precursors with just one block of the polymer. These approaches were inspired by earlier works where charged small-molecule surfactants organized material precursors [16-18] and gradually advanced to include non-charged surfactants [19] and analogous polymers. The family of block polymer SDA approaches grew quickly to include silicates $[14,15]$, metal oxides [20-30], carbonaceous materials, [31,32] and metals [33, 34]. Generally, the block polymer is removed after assembly via a series of heat treatments to yield porous and functional nanomaterials for diverse device applications spanning from absorption, to catalysis and energy devices. The resulting oxide materials generally have surface hydroxyl groups and/or adsorbed water [35-37]. While block polymers have been applied to a wide range of application, e.g., drug delivery [38-40] and porous polymers [41-44], this review article rather focuses upon the recent developments of polymer SDA techniques used to arrange material precursors.

The self-assembly of polymer SDAs generally progresses via free-energy minimization towards specific equilibrium
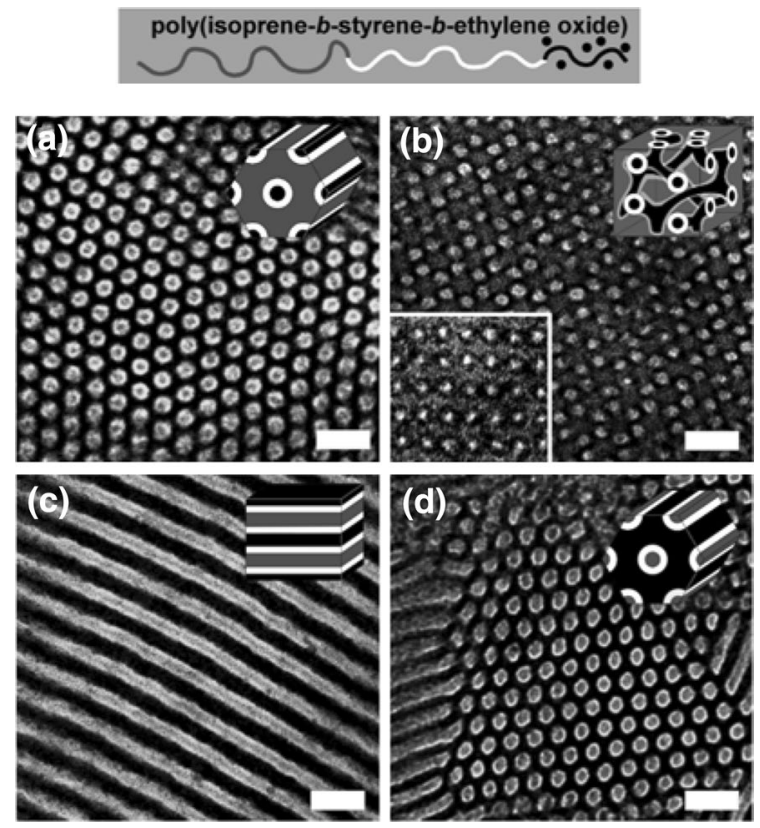

Figure 1: TEM images of different bulk-type polymer morphologies resulting from a poly(isoprene- $b$-styrene- $b$-ethylene oxide) SDA combined with aluminosilicate or niobium oxide material precursors. The morphologies include core-shell hexagonal (A), core-shell double gyroid (B), three-domain lamellae (C), and inverse core-shell hexagonal (D). The scale bars are $50 \mathrm{~nm}$ and the dark regions correspond to the oxide and the $\mathrm{OsO}_{4}$ strained poly(isoprene). Reprinted with permission from Stefik et al. [49]. Copyright 2009 American Chemical Society. 
arrangements. For pure block polymers ("bulk" polymers), these morphologies include motifs such as lamellar sheets, hexagonally arranged cylinders, cubically arranged spheres, continuous gyroids, and other more exotic configurations $[13,45]$. There are both enthalpic and entropic contributions to the free energy. The effective interaction parameter $\left(\chi_{\mathrm{A}-\mathrm{B}}\right)$ is a largely enthalpic term that corresponds to the interfacial energy associated with an interface between two components, e.g., the interface between blocks $\mathrm{A}$ and $\mathrm{B}$. This enthalpic term favors configurations that minimize the total interfacial area, thus promoting larger feature sizes. In contrast, entropic losses from chain stretching favor less-stretched polymer configurations with smaller feature sizes. The balance of these and other free-energy contributions leads to a specific equilibrium state that is either an ordered morphology or a disordered melt. Often this pure block polymer behavior is mapped as a function of $\chi \mathrm{N}$, where $\mathrm{N}$ is proportional to the degree of polymerization, and the volume fraction of a block $\left(f_{\mathrm{A}}\right)$ [46]. One of the distinguishing characteristics of the concept of polymer SDA coassembly is that the addition of material precursors contributes to the free-energy balance and can alter the equilibrium morphology as compared to the pure block polymer itself [47-49]. This is most plainly apparent by recognizing that the selective association of material precursors with one block effectively increases the volume fraction, $f_{\mathrm{A}}$, of that block and can thus change the equilibrium morphology. Figure 1 presents such an example where altering the quantity of material precursors leads to transitions between several ordered morphologies, including hexagonal, lamellar, and gyroid [49]. Here, this is termed bulk-type SDA behavior and is analogous to the equilibrium morphology diagrams of pure polymers.

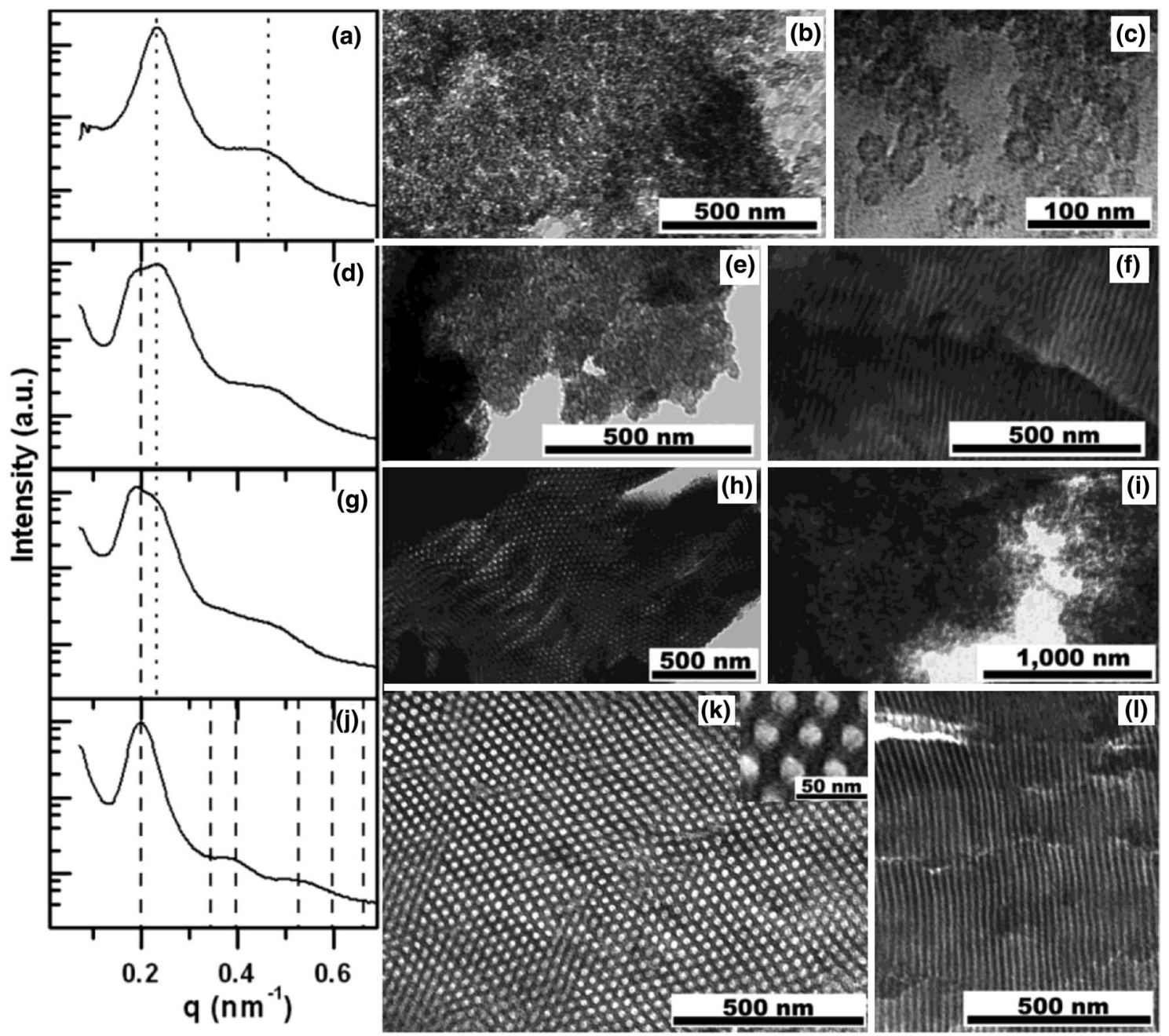

Figure 2: SAXS and TEM data for a poly(isoprene-b-ethylene oxide) SDA combined with $\mathrm{TiO}_{2}$ precursors that transitions between bulk-type (bottom row) and solution-type behaviors (top row). The inverse hexagonally arranged cylinder morphology ( $\mathrm{j}-\mathrm{l}$ ) is a bulk-type behavior that resulted from low solvent selectivity (THF with $1.08 \mathrm{wt} \%$ water). The micelle morphology $(\mathrm{a}-\mathrm{c})$ is a solution-type behavior resulting from moderate solvent selectivity (THF with $4.0 \mathrm{wt} \%$ water). Intermediate behaviors spanning these conditions are shown for $2.0 \mathrm{wt} \%$ water $(\mathrm{d}-\mathrm{f}$ ) and $3.0 \mathrm{wt} \%$ water ( $\mathrm{g}-\mathrm{i})$. The dotted lines correspond to the micellar morphology (a) and the dashed lines correspond to the hexagonal morphology (j). Reproduced from Stefik et al. Ref. [52] with permission from the Royal Society of Chemistry. 


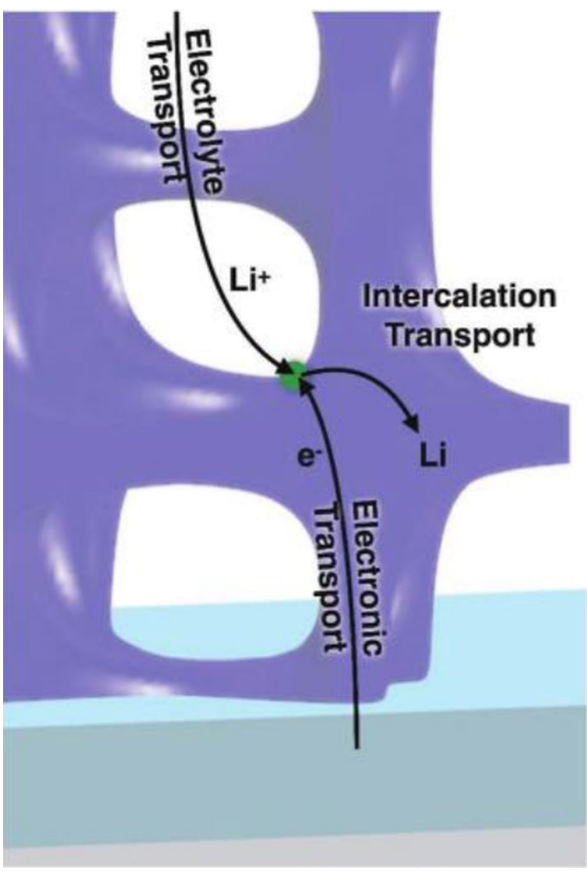

Figure 3: The charging/discharging of a battery electrode involves three concomitant diffusive transport processes (black arrows) as well as an ion insertion/extraction step at a surface (green circle). The study of individual steps is challenging since all these processes are convolved during electrochemical measurements of current/voltage. Improved synthesis methodologies that allow the adjustment of a single nanostructure parameter at a time (pore size, wall thickness, or film thickness) elucidate relationships for the associated properties and performance. Reproduced from van den Bergh et al. from Ref. [55] with permission from John Wiley and Sons.

Materials science traditionally recognizes the importance of processing pathways upon outcomes with, e.g., time-temperature-transformation (TTT) diagrams. Analogously, polymer SDA processing is also dependent upon a range of conditions and is similarly affected by kinetics and transformation rates $[50,51]$. For example, the trace water associated with popular material precursors significantly alters the selectivity of the solvent phase towards the hydrophobic block. In other words, trace water generally increases the interaction parameter between the hydrophobic block and the solvent $\left(\chi_{\text {core-solvent }}\right)$ and thus often induces micellization of the polymer chains with the hydrophobic block residing in the core of the micelles, termed solutiontype behavior. The final morphology is naturally affected by the time and kinetic rates with which the block polymer micelles transition towards the classic morphologies of bulk polymers, i.e., transition from solution-type to bulk-type behavior [52]. This continuum was shown to be controlled by solvent selectivity where minimization of the trace water content leads to bulk-type behavior and iterative increases in water content preserved monotonically more dynamic micelle content (Fig. 2) [52]. These examples vary only in the content of trace water during evaporative processing, highlighting the critical role of (a) Block Polymer Coassembly (Bulk-type)

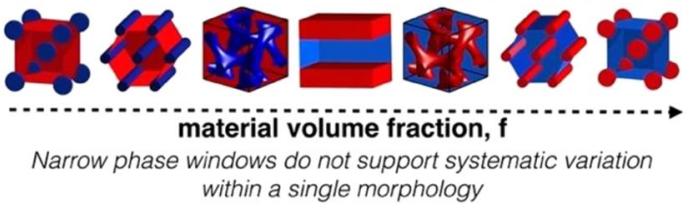

(b) Persistent Micelle Templates (Solution-type)

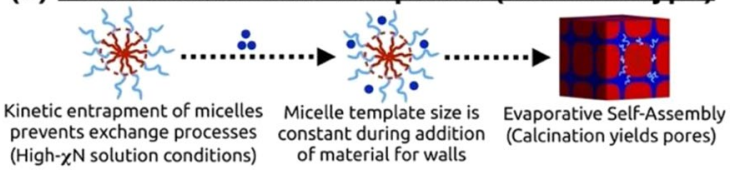

isomorphic, tunable walls, constant pore size

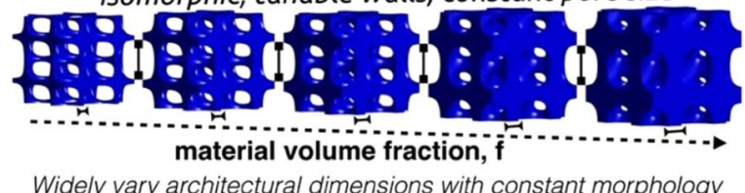

(c) Polymer SDA Continuum of Behavior

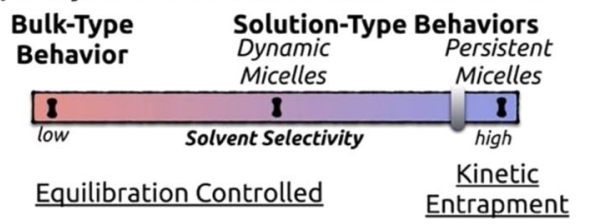

Figure 4: Bulk-type polymer SDA behavior uses equilibration to enable materials with diverse morphologies where numerous attributes vary with the addition of material precursors (a). In contrast, persistent micelle templates use kinetic micelle control and enable sample series with constant morphology and constant pore size with independent control of the wall thickness (b). Solvent selectivity determines the SDA behavior (c) and whether the architectural dimensions are controlled by equilibration (bulk-type and dynamic micelles) or kinetic entrapment (persistent micelles).

processing conditions upon the self-assembly outcomes. Here, the changes in $\chi_{\text {core-solvent }}$ affect the final nanostructure, analogous to temperature control on a TTT diagram. Furthermore, other kinetic aspects such as the material precursor stability [53], their mobility as a function of humidity [21], and their subsequent thermal cross-linking during "aging" treatments [54] prior to crystallization are fundamental to practitioners in this space.

Porous nanoscale materials derived from polymer SDAs are poised to elucidate novel nanostructure-property-performance relationships. As anyone in the sciences can attest, experiments are ideally carried out by changing a single independent variable at a time with as many control (constant) parameters as possible. Despite clear utility and interest, such independent control over spatial parameters from polymer SDAs has remained elusive. Consider, for example, the case of a battery electrode (Fig. 3) [55]. Like other electrochemical devices, its operation involves the concomitant transport of electrons, the transport of ions, and the interconversion between reduced and oxidized states 
(e.g., intercalation). The example of a battery electrode has three transport processes occurring along distinct pathways as pictured. From an electrochemical kinetics perspective, however, those three diffusive processes all have the same rate dependence upon the square root of time, often making it ambiguous as to which specific process is identified by a given measurement. This is a fundamental challenge that is present for diverse electrochemical techniques, including cyclic voltammetry, electrochemical impedance spectroscopy [56], 3D bode analysis [57], and related approaches. Nanostructure-dependent behaviors are better studied with careful nanostructure alterations. PMT enables the production of multiple samples with a single altered spatial variable that can reveal nanostructure cause-and-effect. Unfortunately, strategies that include polymer equilibration determine the final architecture via free-energy minimization where the pore size, the wall thickness, and the overall morphology are all simultaneously sensitive to synthetic variations. Some have called such behavior the "tyranny of the equilibrium" [58] since it hampers access to useful alternatives [59]. Both the bulk-type and solution-type behaviors described above can dynamically progress towards an equilibrium thus convolving changes to multiple architectural parameters (Fig. 4c). With bulk-type behavior, the addition of further material precursors often changes the observed morphology (Fig. 1) which then obfuscates electrochemical study due to the altered tortuosity [60-62]. Similarly, dynamic micelles (solution-type behavior) change the micelle diameter in response to solution composition changes. Thus, when one adds further material precursors to dynamic micelles both the pore size and wall thickness change simultaneously (see Fig. 7 in [63]). A unique approach to sidestep these limitations of equilibrating approaches is to rather rely upon kinetic entrapment (Fig. 4c).

\section{Persistent micelle templates}

Nanomaterials may be produced using PMTs where there is independent control over one spatial parameter at a time [12, 64]. Departing from the convention of processing with equilibration, PMT rather relies upon kinetic entrapment of polymer micelles. As noted above, the limitation of dynamic micelles is that the size can change in response to any alteration of the solution thermodynamics (e.g., composition changes) where active chain exchange mechanisms allow each micelle to alter the aggregation number (the number of chains per micelle) and thus change the overall micelle size. For PMT, these chain exchange processes are inhibited such that the micelle templates have a persistent aggregation number and thus have a constant micelle core size that results in constant pore size after use as a template. Thus, when persistent micelles are used as templates, the addition of material precursors determines the final material wall thickness alone (Fig. 4b) [12]. This independent control enables PMT methods to produce unique sample series with constant pore size and independently varied wall thickness. Furthermore, the preservation specifically of spherical micelles during PMT, rather than transitioning to bulk-type morphologies, enables the production of isomorphic (constant morphology) sample series owing to the flexibility of simple sphere packing. Persistent micelles thus behave as templates in the most literal sense of the word since the template dimension is invariant during the pattern formation process. It should be noted that other block polymer approaches have claimed independent control of pore size or wall thickness earlier, however, those approaches did not consider kinetic micelle size control and did not have predictive models [65-69]. This review paper thus focuses on the development of PMT and how its ability to produce singlevariable porous nanomaterial series enables the identification of nanoscale cause-and-effect relationships.

The kinetic control of micelle size in PMT is based upon the suppression of micelle chain exchange mechanisms such as single chain exchange or fusion/fission. The kinetic rates of single chain exchange have a mature and quantitatively accurate model where the rate of chain exchange has a double exponential dependence upon both the chain length (proportional to $\mathrm{N})$ and the effective interaction parameter between the core block and the solvent phase $\left(\chi_{\text {core-solvent }}\right)$. [70-72] This very stiff function has been described as being hypersensitive to changes in chain length [70], however, the same model naturally also predicts hypersensitivity to $\chi_{\text {core-solvent }}$. These dependencies reflect that the rate-limiting step of single chain exchange is the extraction of a core block from a micelle where its dispersion requires energy corresponding to the thermodynamic cost of the newly formed interface $\left(\chi_{\text {core-solvent }}\right)$ and its size (scales with $\mathrm{N}$ ). Both single chain exchange and fusion/fission chain exchange mechanisms are dependent upon the solvent selectivity [50, 73-76]. The dependence of PMT control upon solvent selectivity was revealed in the first PMT publication [12]. A series of samples was produced from a poly(ethylene oxide- $b$-hexyl acrylate) (PEO- $b$-PHA) with $63.5 \mathrm{~kg} / \mathrm{mol}$ (P3) using different amounts of material precursors. The varying amount of material precursor is noted using the material:template ratio (M:T). The processing solvents were a mixture of a good solvent for the overall polymer, tetrahydrofuran (THF), and a poor solvent for the PHA core block, water. The two samples with the lowest M:T ratios (P3-1.2 and P3-2.4) had statistically indistinguishable average pore sizes and the sample with the highest M:T ratio had the largest average wall thickness (Fig. 5a, b, d, e). In contrast, however, the addition of further material precursors to sample P3-3.0 resulted in a decreased average pore size, clearly revealing a transition to the active chain exchange that is characteristic of dynamic micelles (Fig. 5 c, d). This observation 

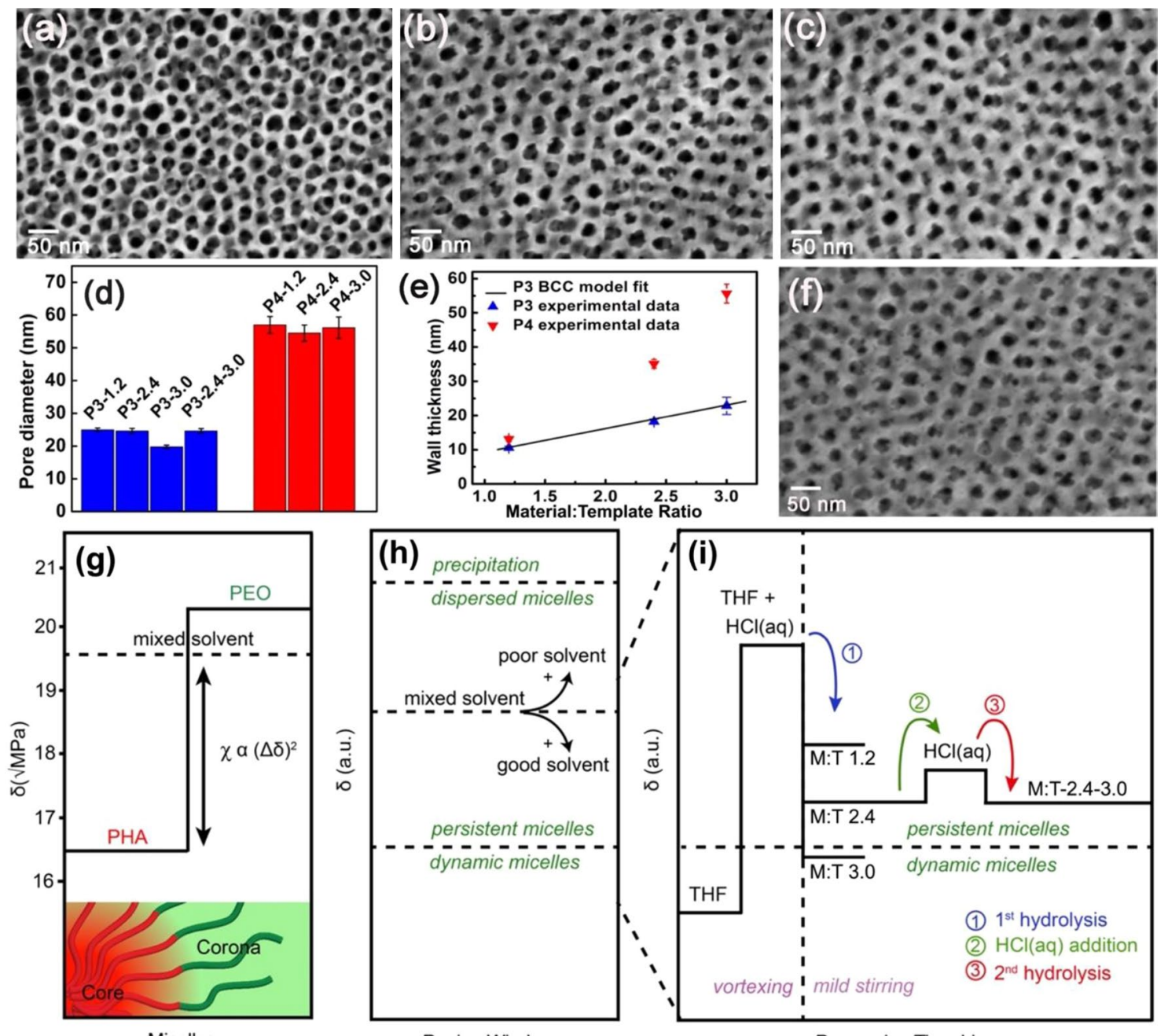

Design Window

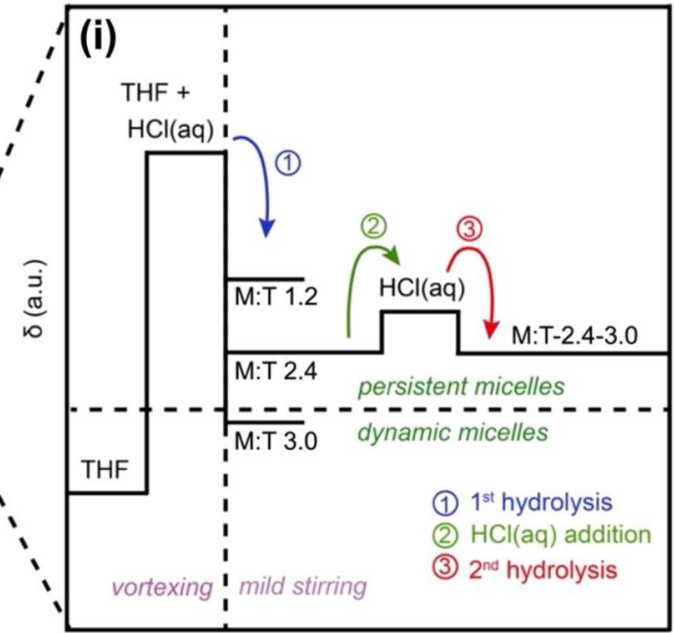

Processing Time Line

Figure 5: Samples prepared from a poly(ethylene oxide-b-hexyl acrylate) " $\mathrm{P} 3$ " $\mathrm{SDA}$ with different material:template ratios using $\mathrm{Nb}_{2} \mathrm{O}_{5}$ precursors (a-c). The decrease in pore size upon reaching M:T $=3.0(c, d)$ corresponded to the transition from persistent micelles to dynamic micelles. This behavior change is predicted by solvent selectivity as implied by plots of Hildebrand solubility parameters $(\mathrm{g}$ ) where the separation between the core block and the solvent mixture adjusts the enthalpic barrier to chain exchange. A range of behaviors is anticipated depending on the amount of good vs poor solvents, i.e., the solvent selectivity (h). Processing micelles entirely within the persistent micelle window (i) enables a series with constant pore size (d, f) and independently varied wall thickness (e). Reprinted with permission from Lokupitiya et al. [12]. Copyright 2016 American Chemical Society.

revealed the first modality of PMT control. Here, it is important to recognize that the material precursors are not benign towards the solvent composition. This set of samples produced porous $\mathrm{Nb}_{2} \mathrm{O}_{5}$ from niobium ethoxide, where such alkoxides are known to strongly react with water via hydrolysis [77]. These changes in water composition directly change the enthalpic $\chi_{\text {core-solvent }}$ barrier to chain exchange.

Changes in $\chi$ parameters may be crudely estimated using Hildebrand solubility parameters $(\delta)$ where $\chi \alpha\left(\delta_{\text {core }}-\delta_{\text {solvent }}\right)^{2}$ [78]. Hildebrand solubility parameters scale with the strength of intermolecular interactions and are volume-normalized. Though interaction parameter estimations from solubility parameters are semi-quantitative at best, they do scale monotonically with reality which makes them a distinctly convenient tool. It follows that the interaction parameter $\chi$ scales with the distance between two entities in Hildebrand space (Fig. 5g). A range of polymer behaviors are expected depending on the $\chi_{\text {core-solvent }}$ value, where lower values lead to dynamic micelles with active chain exchange and higher values lead to persistent micelles without chain exchange (Fig. 5h). The Hildebrand parameter for solvent mixtures is simply the volume-weighted average of the constituent solvents. Thus, the $\chi_{\text {core-solvent }}$ is easy to adjust: the addition of good solvents for the core block decreases $\chi_{\text {core-solvent }}$, whereas the addition of poor solvents for the core block increases $\chi_{\text {core-solvent }}$ (Fig. $5 \mathrm{~h}$ ). This provides a simple conceptual framework for experimentalists to adjust micelle thermodynamics and kinetics. Returning to the dynamic micelle behavior of sample P3-3.0, now may be understood where the 

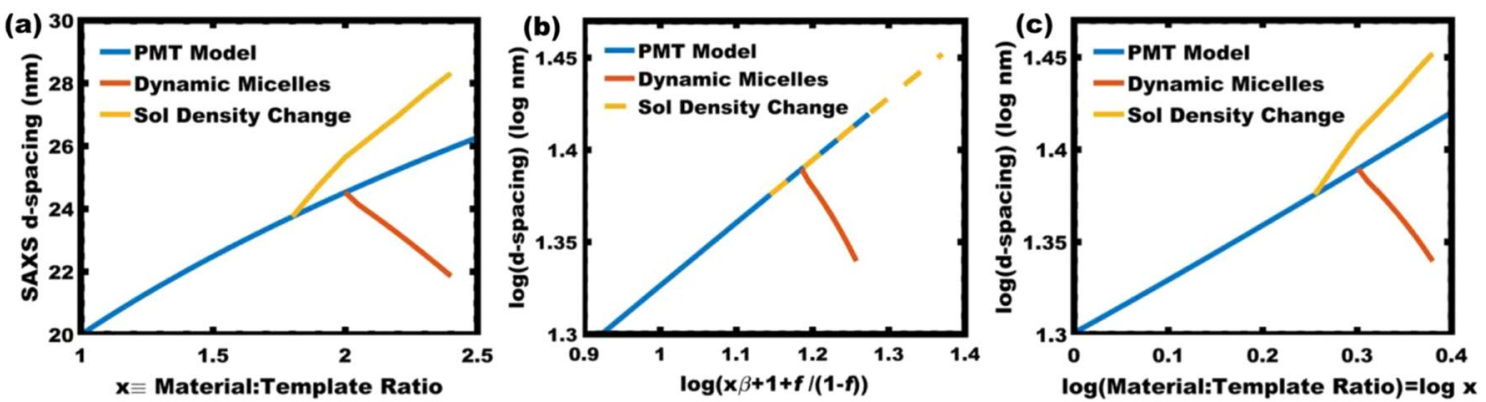

Figure 6: SAXS d-spacing expectations predicted by a PMT model where increasing Material:Template ratio ( $x$ for brevity) causes lattice expansion (a). The use of this model requires knowledge of the average pore size from other measurements. Deviation from the model indicates either a loss of kinetic micelle control or violation of one of the model assumptions (yellow and orange lines). A log-log rearrangement enables model usage without knowledge of the pore size, however, this requires a priori knowledge of the $\beta$ fit-term value (b). Further rearrangement and an approximation yield a convenient form where PMT sample series may be checked for consistency with PMT behavior (approximate slope of 1/3) using SAXS data alone (c). Reprinted with permission from Sarkar et al. [81]. Copyright 2018 American Chemical Society.

addition of material precursors to aqueous THF monotonically reduced the water content and decreased the enthalpic barrier to chain exchange (Fig. 5i). Now that the behavior is understood, kinetic control may be restored with an experimental adjustment: Sample P3-2.4-3.0 was prepared analogous to P3-2.4, however, additional aqueous $\mathrm{HCl}$ was added prior to the addition of further alkoxide material precursors. When walking this trajectory through Hildebrand space one may continuously stay within the persistent micelle regime and thus preserve micelle kinetic control (Fig. 5i). This seminal study thus established the foundational principles of PMT control based upon solvent selectivity. [12, 64].

Again analogous to TTT diagrams, the complete polymer SDA processing path influences the outcomes with all of the polymer SDA behaviors described thus far (Fig. 4). In particular, solvent selectivity plays a central role on the continuum of SDA behaviors, as elaborated above. It is important to recognize that solvent selectivity changes throughout evaporative processing where boiling points and azeotropes should be considered $[79,80]$. Under the general case of selective solvent conditions there will be micelles present during the evaporation process. The subsequent behavior continuum with solvent selectivity is summarized in Fig. 4c where low solvent selectivity enables bulk-type SDA behavior and increasing solvent selectivity preserves solution-type behavior. Within the realm of solution-type behaviors there are two further regimes. Moderately selective conditions yield dynamic micelles where the resulting pore size can vary with any change to the solution composition. This thermodynamic response is why both bulk-type behavior and dynamic micelles are best termed as coassembly since the outcome is influenced by equilibration. In contrast, high solvent selectivity leads to persistent micelles where their size does not change in response to shifting equilibrium conditions. Thus, the phrase "template" is most appropriate for PMT since its character is invariant to diverse processing conditions.

\section{Easy validation and PMT titration}

A simple geometric model was developed to identify consistency with PMT behavior via convenient small-angle X-ray scattering (SAXS) measurements [63]. The first PMT demonstration required hundreds of manual measurements on SEM images to derive statistically significant metrics of average pore size and average wall thickness for each sample. In contrast, X-ray scattering measurements of such materials directly correspond to average unit cell dimensions across the few $\mathrm{mm}^{2}$ measurement area. Thus, a model was developed to relate the unit cell dimensions to the micelle-to-micelle spacing and then deconvolve that into the underlying pore size and wall thickness. The deconvolution is based upon a simple conservation of volume argument where the amount of micelles and the amount of material precursors are known for each sample via the M:T ratio. For PMT conditions, the addition of material precursor volume will increase the distance between neighboring micelles, thus resulting in lattice expansion. Quantitative models for lattice expansion were derived for all common cubic unit cells as well as a generalized model for paracrystals containing disorder [63]. All of the resulting models had a common quasi-cube root dependence of SAXS d-spacing upon the M:T ratio, reflecting the fundamental relationship of a linear dimension to a volumetric term (Fig. 6a). The primary assumption of the model is a constant structure factor that implies a proportionality between micelle-to-micelle spacing and the observed structure factor peak by SAXS. A deviation from the PMT model generally indicates a change in the micelle size, signaling a transition towards dynamic micelles, or could also arise theoretically from a change, e.g., in the material density. Fitting this PMT model to SAXS data, however, required real-space measurements for at least one sample, e.g., from SEM measurements. A useful log rearrangement of the PMT model eliminates this need for real-space data for the assessment of consistency with 

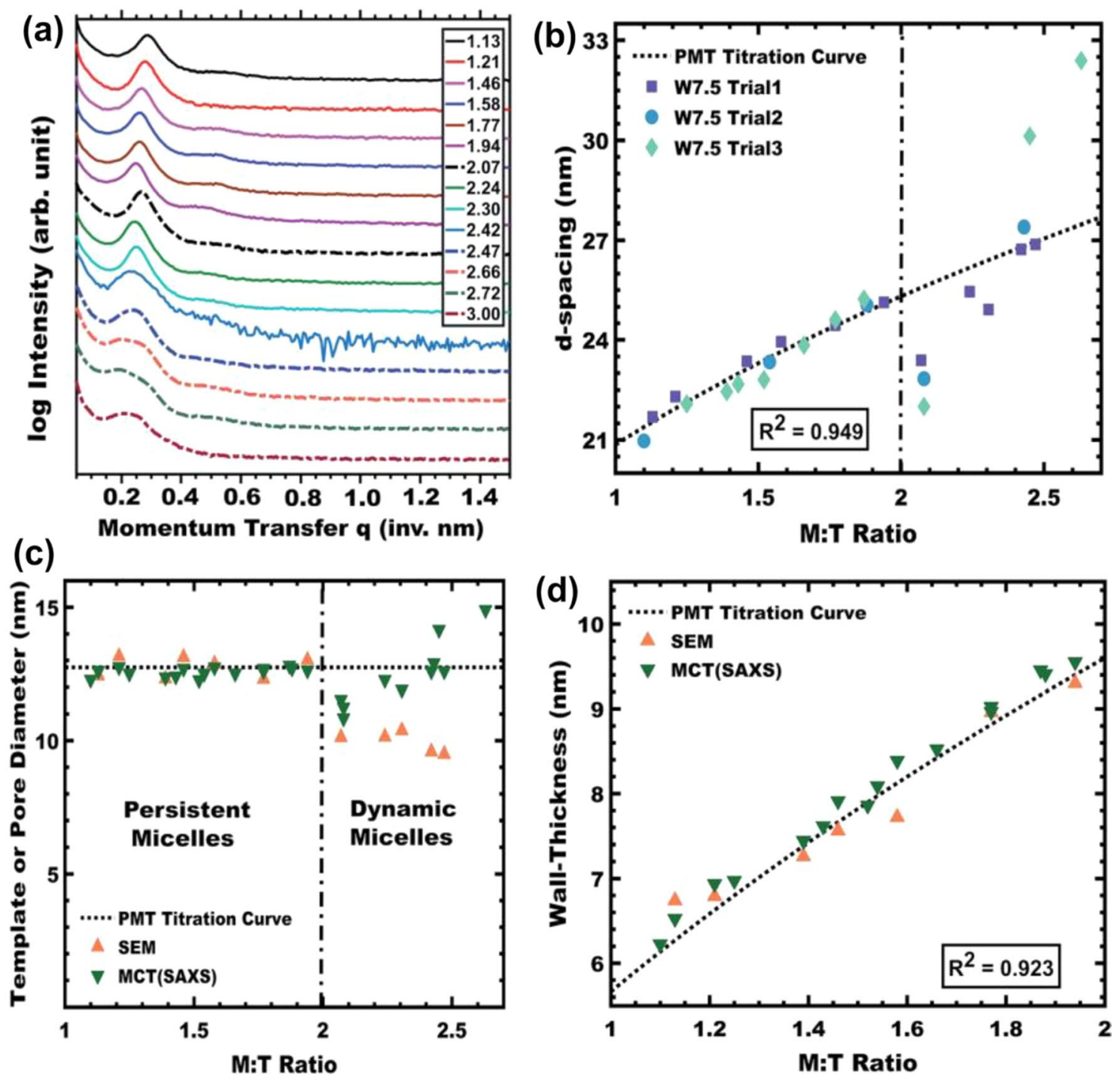

Figure 7: SAXS data from a PEO- $b$-PHA SDA combined with $\mathrm{Nb}_{2} \mathrm{O}_{5}$ material precursors via titration (a). The increasing d-spacing corresponds to the lattice expansion predicted by the PMT model (b). Model fitting enables extraction of the underlying average template/pore diameter (c) as well as the average wall thickness (d). The model interpretations (c, d) of SAXS data (green) closely match direct model-independent measurements by SEM (orange). Reproduced from Sarkar et al. from Ref. [63] with permission from the Royal Society of Chemistry.

PMT conditions [81]. Here, a straight line with slope of $1 / 3$ is consistent with PMT conditions, however, the corresponding $x$-axis requires a priori knowledge of the $\beta$ value, a convolved density fit term (Fig. 6b). Further algebraic rearrangements and an approximation yield a more utilitarian relationship where a plot of $\log (\mathrm{d}$-spacing $)$ vs $\log (\mathrm{M}: \mathrm{T})$ yields a slope of approximately $1 / 3$ for PMT behavior (Fig. 6c). This latter scaling relationship conveniently enables a sample series to be checked for consistency with PMT behavior using SAXS data alone prior to real-space measurements such as electron microscopy.

An easy titration method was recently developed for producing PMT sample series from a single vial of micelle solution [63]. Here, an M:T series is iterated by adding material precursors to the micelle solution followed by spin coating samples in a repetitive fashion. SAXS measurements from such titration series reveal the SAXS peak shifting to smaller values in reciprocal space, corresponding to lattice expansion in real-space (Fig. 7a, b). The data from M:T 1-2 were well fitted by the PMT model with a goodness-of-fit $R^{2}$ of 0.949 . Higher M:T ratios led to the same transition to dynamic micelle behavior explained above based upon poor-solvent (water) depletion. Within the identified window of PMT conditions, the pore size and wall thickness are well predicted by the PMT model and were quantitatively similar to direct real-space measurements by SEM (Fig. 7c, d). For example, the changes in wall thickness had a goodness-of-fit $R^{2}$ of 0.923 . Also noteworthy here are the 16 samples spanning a $2.56 \mathrm{~nm}$ change in average wall thickness that corresponds to nominal resolution of a $\sim 2 \AA(0.2 \mathrm{~nm})$ 
increment of adjustment between samples. This precision control over the average wall thickness is commensurate with the atomic dimensions of the atoms within the $\mathrm{Nb}_{2} \mathrm{O}_{5}$ walls. It should be noted that each of these samples has a distribution of wall thickness values where neighboring samples have overlapping distributions when using such small and precise adjustments to the mean value.

\section{Expanded PMT versatility}

The width of the PMT window determines the range of architectural dimensions that may be accessed with independent wall thickness control (Fig. 7c, d). The early PMT studies identified a critical role of water upon the width of the PMT window (Fig. 5i) [12]. However, the use of water alone to expand PMT windows is not an ideal proposition because beyond a certain threshold the water itself introduces other problems where it can phase separate and produce random, uncontrolled porosity [81]. Since the enthalpic barrier to chain exchange depends upon the mixture of solvents, a new strategy was developed where the majority solvent was changed to increase the baseline Hildebrand solubility parameter prior to the addition of water (Fig. 5g) [81]. That study compared methanol (MeOH, $\left.\delta_{\mathrm{MeOH}}=29.7 \sqrt{\mathrm{MPa}}\right)$ and ethanol $\left(\mathrm{EtOH}, \delta_{\mathrm{EthOH}}=26.2 \sqrt{\mathrm{MPa}}\right)$ to THF $\left(\delta_{\mathrm{THF}}=18.5-19.3 \sqrt{\mathrm{MPa}}\right)[78,82]$. DLS measurements of such solutions alone can identify dynamic micelles by the observation of free chains in solution, however, due to the sizedependent scattering strength it should be noted that the lack of observed free chains is not itself sufficient evidence of persistent micelles. Indeed, despite the lack of observable free PEO- $b$-PHA chains in $\mathrm{MeOH}$ or EtOH by DLS the subsequent PMT titrations for each revealed a change in pore size when the trace water became depleted via hydrolysis. Despite the need for trace water, a remarkable $3 \times$ increase in the PMT window width was found when switching the majority solvent from THF to these alcohols. Contrary to expectations from solubility parameters alone, however, both $\mathrm{MeOH}$ and $\mathrm{EtOH}$ led to similar PMT window widths, within the uncertainty of the experiment. The suggested causes for this observation included differences in viscosity, changes in alkoxide kinetics after alcohol exchange with the solvent, and solvent-dependent rates of hydrolysis. These considerations highlight the unintended materials chemistry complexities that can result from seemingly simple alterations.
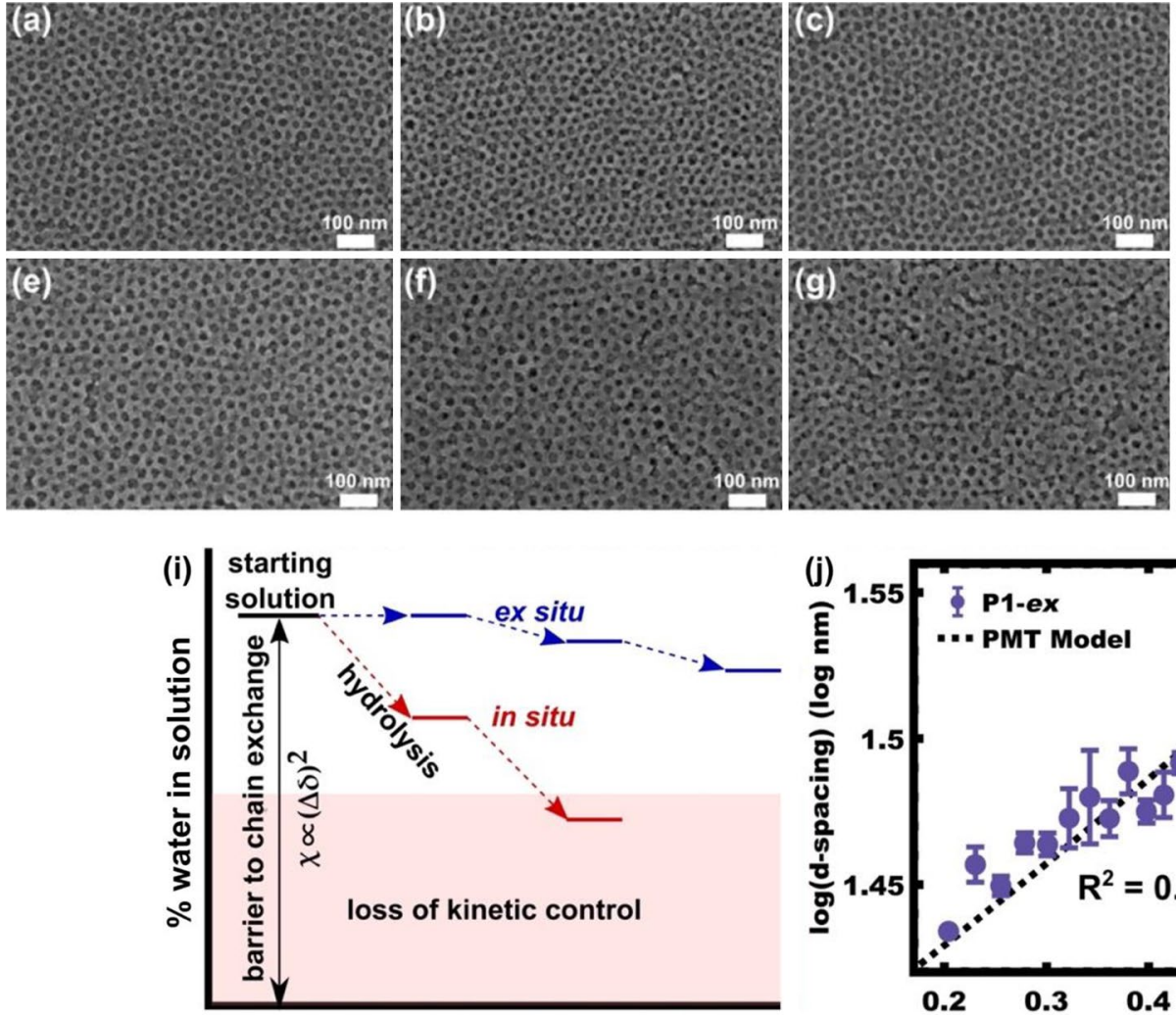

Material:Template Ratio

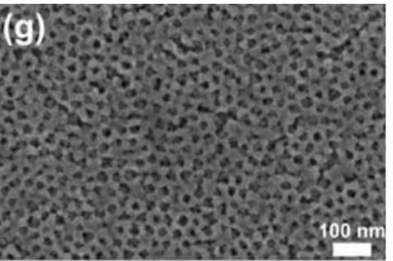

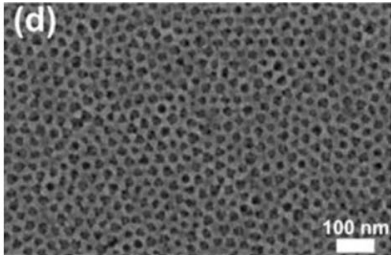
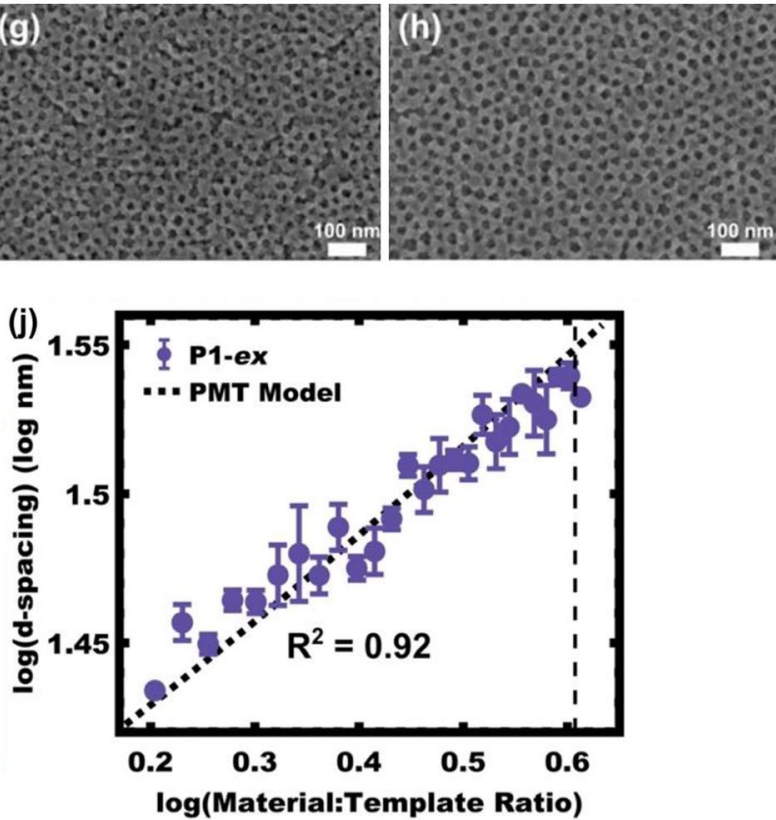

Figure 8: An ex situ approach for material precursor hydrolysis was combined with PEO- $b$-PHA PMTs to reduce the coupling between material additions and micelle persistence (i). A series of $25 \mathrm{TiO}_{2}$ samples with different Material:Template ratios were consistent with PMT behavior by SEM and SAXS (j). The SEM images correspond to M:T=1.6 (a), 2.0 (b), 2.3 (c), 2.7 (d), 3.0 (e), 3.4 (f), 4.0 (g), and 4.1 (h). Adapted from Lantz et al. from Ref. [83] with permission from John Wiley and Sons. 
Regardless, alcohols were shown to be the most ideal solvents for PMT processing to date.

The coupling of material additions to a loss in kinetic micelle control were largely relaxed by the implementation of ex situ hydrolysis [83]. For the earlier PMT demonstrations, raw alkoxides were added to aqueous persistent micelle solutions to increase the M:T ratio in an in situ fashion. That approach, however, necessarily depleted the water content, and thus depleted the barrier to chain exchange with each material addition (Fig. 8i). In contrast, ex situ hydrolysis accomplishes the same reaction in a separate solution. The use of ex situ hydrolysis thus largely decouples micelle kinetic control from the later addition of material precursors (Fig. 8i). The corresponding M:T series enabled the demonstration of the widest PMT window ( $\mathrm{M}: \mathrm{T}=1.6-4.0)$ to date with 25 samples spanning the gamut from sparse walls to nearly isolated pores (Fig. 8). It should be noted that the ex situ hydrolysis development itself required an optimization of water content to account for both hydrolysis reactions which consume water and condensation reactions that release water. For example, a 4:1 ratio of water to titanium isopropoxide led to film dewetting from the substrates due to excessive water where a 3:1 ratio was sufficient to preserve micelle kinetic control without film dewetting. Thus, the deployment of ex situ hydrolysis was shown to largely decouple materials chemistry from micelle kinetic control.

For a given block polymer, the range of accessible persistent micelle sizes and thus the range of accessible pore sizes was greatly expanded by the introduction of homopolymer swelling [84]. The ability of homopolymers to swell the analogous block of a block polymer is thoroughly established in both the contexts of bulk-type behavior and solution-type behavior. A typical guideline is that the homopolymer should have a lower molar mass than the block it swells on the block polymer. Following this and further guidelines [84], a poly(hexyl acrylate) homopolymer was combined with PEO- $b$-PHA micelles and was shown to enable up to $1.3 \times$ pore diameter increase while maintaining a monomodal pore size distribution with up to $250 \mathrm{wt} \%$ homopolymer, considerably higher than the $~ 90 \mathrm{wt} \%$ limit found for dynamic micelles $[85,86]$. With the PMT process often being conducted near the borders of micelle persistence (Fig. 5i), the decreased molar mass of the homopolymer (lower $\mathrm{N})$ naturally implies that the homopolymer is likely to exhibit

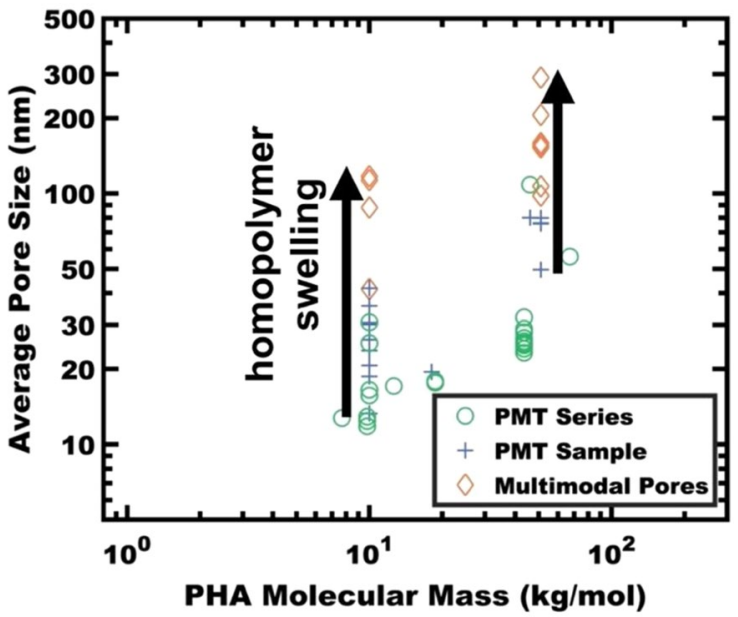

Figure 10: Demonstrated PMT pore sizes to date span from the mesoporous regime $(2-50 \mathrm{~nm})$ to the macroporous regime $(>50 \mathrm{~nm})$. Pore sizes vary with the molar mass of the core block ( $x$-axis), the micelle processing prior to kinetic entrapment (vertical isopleths), and homopolymer swelling (arrows).
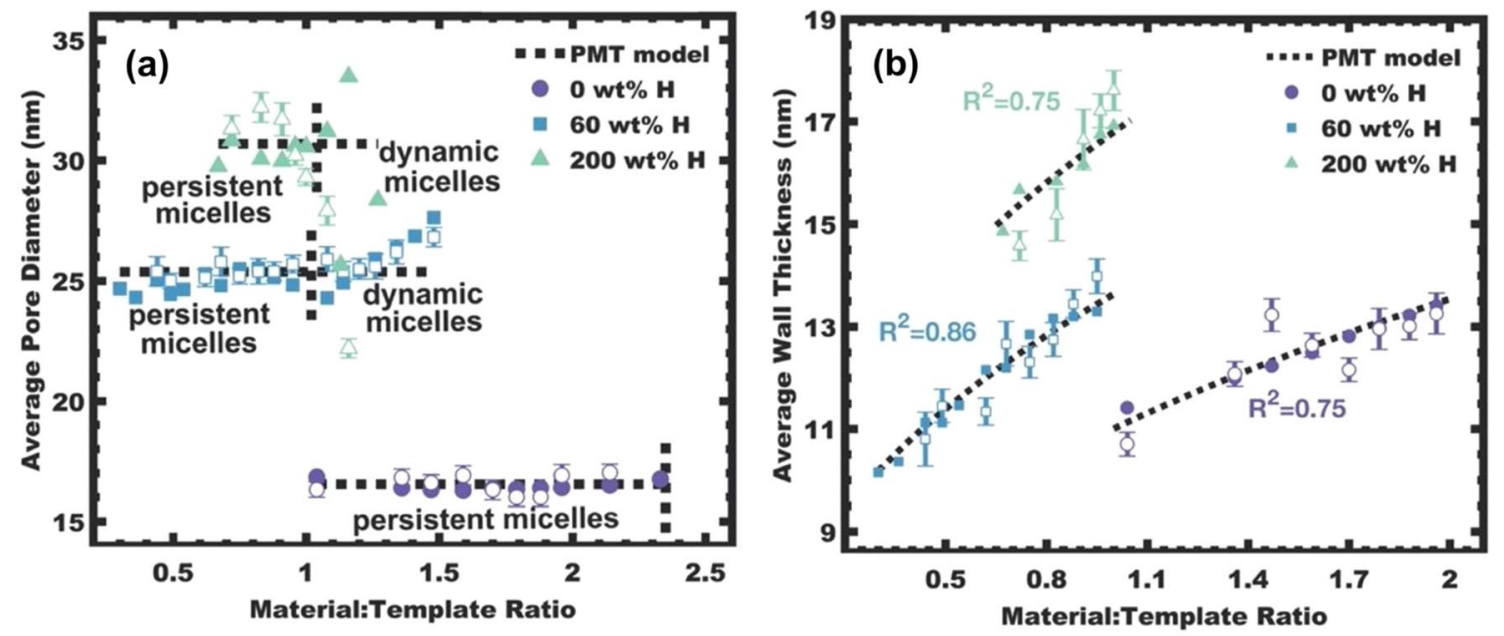

Figure 9: The addition of PHA homopolymer swelling to PEO-b-PHA PMTs enables wide variation of the micelle/pore diameter (a) followed by material titration to independently adjust the wall thickness (b). A single PEO- $b$-PHA block polymer and a single PHA homopolymer were used to produce all of these porous $\mathrm{Nb}_{2} \mathrm{O}_{5}$ architectures. Adapted from Sarkar et al. from Ref. [84] with permission from the Royal Society of Chemistry. 
dynamic exchange between micelles. Indeed, both $\mathrm{MeOH}$ and EtOH solutions of these swollen micelles were found to have limited stability where the homopolymer gradually phase separates, thus confirming active exchange of homopolymer between persistent micelles. Nonetheless, PMT titration series were carried out with different amounts of homopolymers. For each swelling condition, the SEM measurements and SAXS modeling both confirmed isomorphic sample series with constant pore size and varied wall thickness (Fig. 9). Thus, this swollen PMT approach offers a unique combination of precision control with wide tunability. Here, the term "persistent" refers to the kinetic entrapment of block polymer chains as well as the constant average diameter of the swollen micelles despite active homopolymer exchange between those micelles. A pronounced effect of solvent selection was found where the homopolymer was found to phase separate more quickly from $\mathrm{MeOH}$ as compared to EtOH. If this phase separation was purely dependent upon the rate of homopolymer exit from the micelle cores then the opposite trend would be expected based on the enthalpic barrier associated with $\chi_{\text {PHA-solvent }}$. The behavior is understandable by considering the outcomes for a homopolymer that has been extracted to the solvent phase. It can either (1) re-enter a micelle core, (2) remain dispersed in the solvent, or (3) aggregate with other homopolymers, forming a precipitate. The lower $\chi$-value for EtOH both increases the homopolymer solubility and facilitates the re-dissolution of precipitated homopolymer. These considerations are another reminder of the confluence of multiple factors while making seemingly simple modifications to materials chemistry. The addition of homopolymer swelling to PMT now enables one to first select the desired pore size via the amount of homopolymer addition and then independently adjust the wall thickness by the addition of material precursors. Thus, the addition of homopolymer swelling to PMT enabled two distinct synthetic handles to directly control the pore size and wall thickness independently.

The feature sizes obtained by PMT are wide spanning from mesoporous materials to macroporous materials [87]. Figure 10 shows the wide range of PMT pore sizes demonstrated to date, spanning from 11.8 to $109 \mathrm{~nm}$ with monomodal pore size distributions. Considering the simple geometric model [63], there is a coupling between the selected pore size and the achievable range of wall thickness values, where the wall thickness is generally $0.2-1.0 \times$ with respect to the pore size. Thus, Fig. 10 also conveys the demonstrated range of PMT average wall thickness. The $x$-axis in Fig. 10 is the molar mass of the PHA core block which is clearly connected to the resulting pore size. The vertical isopleths, however, demonstrate a range of micelle size tuning based upon the processing history [12]. Also cavitation-induced exchange (CIE) is an enabling concept here where kinetically trapped micelles can temporarily become dynamic during ultrasonic cavitation, undergoing chain exchange until cessation at which point the micelles return to a persistent state $[88,89]$. Lastly, the addition of homopolymer swelling to PMT enables facile and wide spanning pore size adjustments with minimal synthesis (Fig. 10 arrows) and also enables multimodal spongelike pore distributions with large pore sizes demonstrated up to $290 \mathrm{~nm}$. PMT is ideal for diverse nanomaterials research since this single technique spans the wide range of feature sizes necessary for the study of diverse nanostructure-property relationships.

\section{Deployment of PMT in diverse laboratories}

The initiation of persistent micelle templates in a new laboratory has minimal barriers to entry. Access to suitable block polymers is typically the first challenge. The block polymers suitable for PMT must have a sufficient $\chi \mathrm{N}$ barrier towards chain exchange which generally requires custom block polymers that are not mass produced. Unfortunately, the very popular Pluronic polymers are unsuitable for PMT owing to the low interaction parameter with alcohols and the low molar masses (low N). PEO is the most popular block for selective SDA interactions with material precursors. The composition of the hydrophobic block for PMT is not particularly crucial so long as there is sufficient barrier to chain exchange. Published PMT demonstrations to date have largely used PEO- $b$-PHA though persistent micelles have also been demonstrated with core blocks composed of poly(styrene) [90], poly(cyclohexyl methacrylate) [91], and poly(n-butyl acrylate). While there are several commercial vendors for suitable block polymers at the gram scale, it should be noted that such polymers are facile to synthesize by those that are not polymer scientists. A recent paper demonstrated that readily available commodity reagents can be used to prepare suitable block polymers from start-to-finish in $20.5 \mathrm{~h}$ using only common glassware, a hot plate, and inert gas (Fig. 11a-m) [63]. Thus, even capitol constrained laboratories are able to prepare, e.g., PEO- $b$-PHA. In brief, the process starts from a commercial PEO homopolymer with (1) an esterification reaction to add an initiation group for atom transfer radical polymerization (ATRP) and (2) subsequent ATRP of the desired second block. Gel permeation chromatography (GPC) and nuclear magnetic resonance spectroscopy (NMR) should be used to validate the uniformity and the block ratios, respectively, which are techniques found on most campuses and are also available as mail-in services. Here, the use of commodity reagents makes it convenient and low cost to prepare 10-100 $\mathrm{g}$ of PMT-suitable block polymer at a time for the sake of convenience.

The preparation of films with PMT control has more subtle challenges with documented and reproducible solutions [63]. A recent publication itemized 18 tips and tricks for successful PMT deployment [63]. For example, the reproducible production of persistent micelles with the same size can be a challenge 
Figure 11: The use of PMTs is easy to conduct in diverse laboratory settings using commodity reagents and minimal equipment. Suitable block polymers can be synthesized with just a hot plate, a nitrogen tank, and common glassware. The complete process of polymer synthesis through spin coating can be completed in less than $24 \mathrm{~h}$ (inset time stamps). The photographs show the sequential process: PEO dissolution (a), esterification of ATRP initiator (b) filtration (c), drying (d), further purification by phase separation (e), removal of oxygen by nitrogen sparging ( $f$ ), polymerization of hexyl acrylate $(g-h)$, removal of copper salts with alumina column $(i, j)$, block polymer precipitation $(k, l)$, and recovery $(m)$. Micelles are prepared with a sonication step (n), material precursors are added via air-free syringe and substrates are coated using a home-made humidity-controlled spin coater $(o-p)$, followed by immediate aging on a hot plate (q), calcination in a furnace ( $r$ ), and finally analysis by cross-sectional (s) and top-view (t) SEM. Adapted from Sarkar et al. from Ref. [63] with permission from the Royal Society of Chemistry.
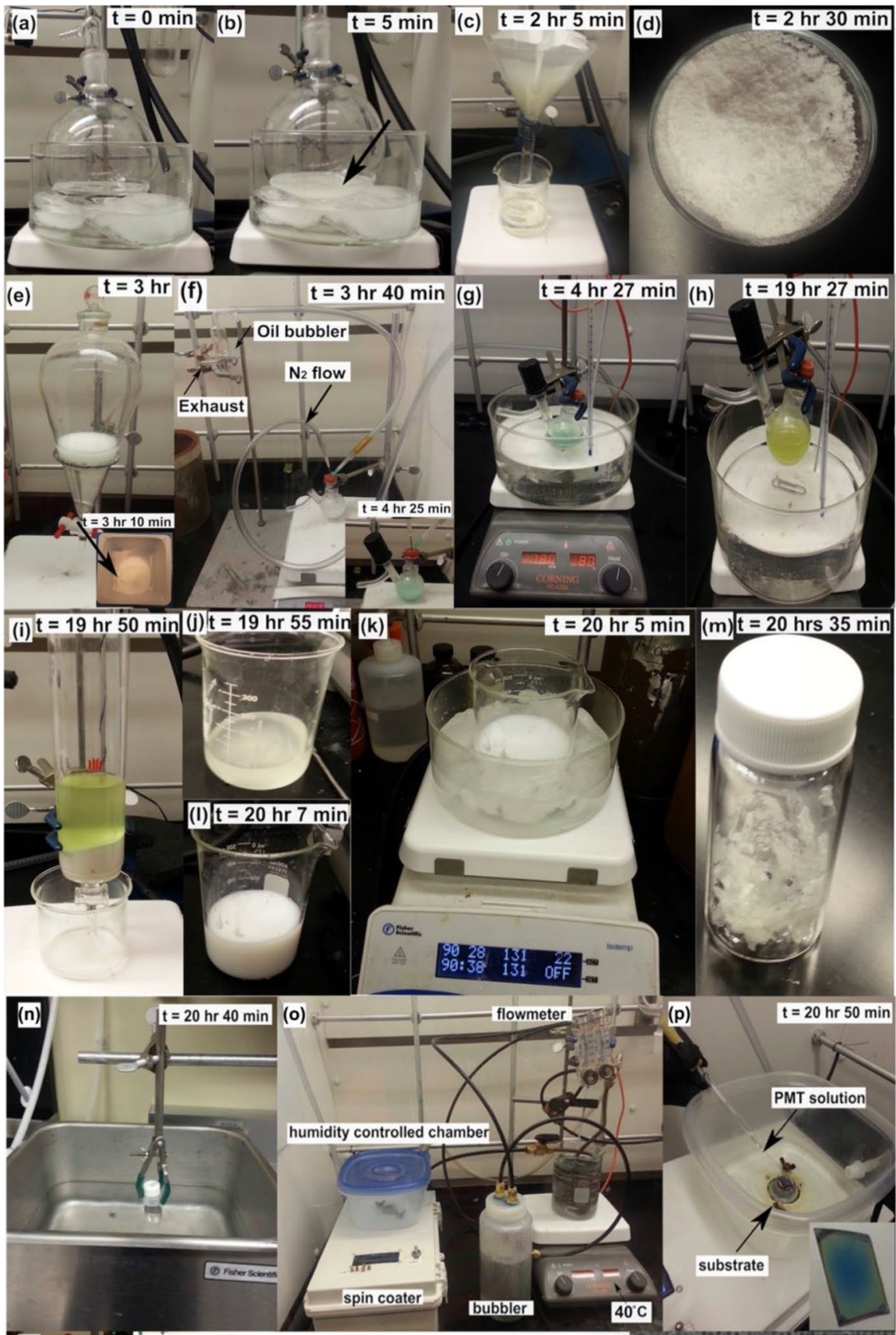

(t)

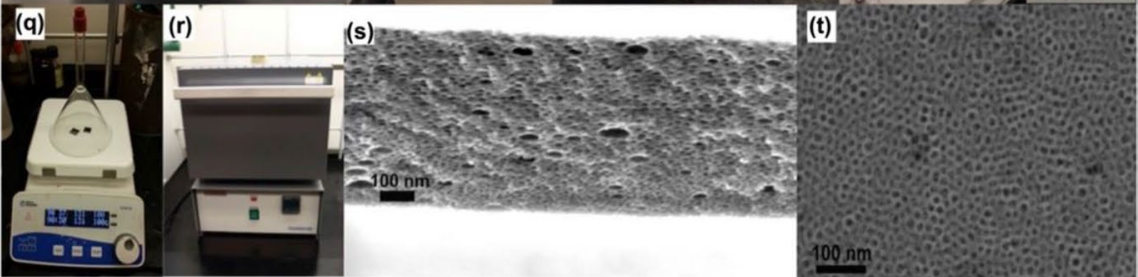

since persistent micelles do not equilibrate. Here, the dropwise addition of the poor solvent to a polymer solution enables reproducible PMT formation. Also the addition of vortexing or ultrasonic cavitation can temporarily activate chain exchange mechanisms for the purpose of micelle size homogenization or size tuning $[88,89,92]$. Figure $7 \mathrm{~b}$ shows the PMT titration curves for three different micelle batches prepared on three different days, demonstrating a high-degree of reproducibility with due diligence. The film formation by spin coating or dip coating, for example, requires an optimized humidity while coating each 

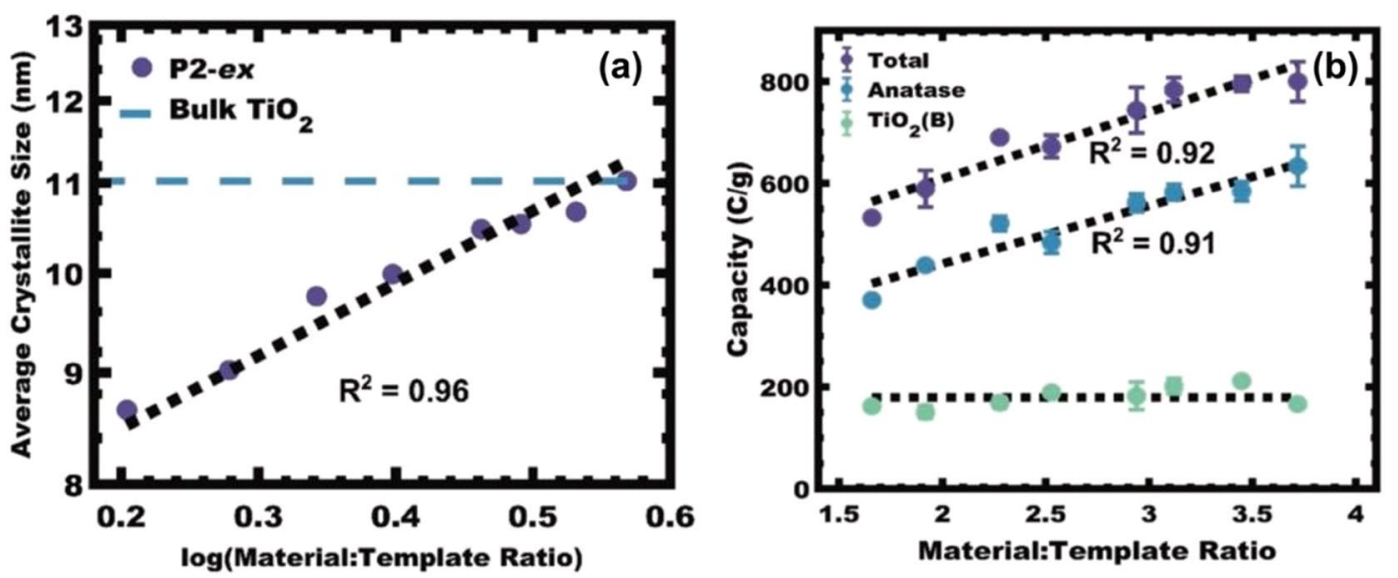

Figure 12: The anatase crystallite size increased with the wall thickness for porous $\mathrm{TiO}_{2}$ samples prepared using PEO-b-PHA PMTs (a). Electrochemical lithiation revealed a second polymorph consistent with bronze phase $\mathrm{TiO}_{2}$. The electrochemical capacity of the apparent bronze phase was constant across the sample series, whereas the anatase capacity increased with crystal size and was attributed to the size-dependent strain mismatch (b). Adapted from Lantz et al. from Ref. [83] with permission from John Wiley and Sons.

material. Although humidity-controlled spin/dip coaters are available, such units are also fairly simple to construct. The interested reader is directed to these DIY instructions for building a humidity-controlled spin coater with vacuum chuck and digital acceleration control using commodity parts [93]. Another subtlety is that spin coating evaporates away the solvent and thus removes the prior barrier to chain exchange. Thus, PMT samples must be immediately moved to a hot plate within a few seconds of drying to have the material precursor cross-linking reaction outrun the micelle reorganization. This "aging" treatment is another typical optimization which has the additional goal of sufficiently cross-linking the material such that the architecture can survive removal of the polymer template and also generally the crystallization of material. The complete PMT process from polymer synthesis through PMT spin coating is pictured in Fig. 11 with time stamps showing that the entire process can be accomplished from scratch using commodity reagents in less than $24 \mathrm{~h}$.

\section{Single-variable studies of nanoscale architectures}

Nanoscale crystalline materials are well known to exhibit sizedependent properties. Towards this end, PMTs provide a highly tunable confinement environment for the controlled crystallization of material precursors. A recent PMT study examining $\mathrm{TiO}_{2}$ crystallization with varied wall thickness identified monotonic control over the resulting average anatase crystallite size ranging from $\sim 8.5$ to $11 \mathrm{~nm}$ (Fig. 12a) [83]. For this set of samples, the average crystallite size was generally about half of the measured wall thickness, showing that the nanostructure confines the crystal growth and suggesting dominant surface nucleation. This sample series was also examined as lithium ion battery anodes. The lithiation trend for specific capacity (massnormalized) monotonically increased with the wall thickness (Fig. 12b). The cyclic voltammetry data also revealed an addition feature consistent with bronze $\mathrm{TiO}_{2}$, suggesting a mixture of polymorphs. Curiously, the electrochemical capacity of the apparent bronze phase was constant across the M:T series, whereas the capacity of the anatase phase monotonically increased with wall thickness. This behavior was interpreted as the result of a sizedependent strain mismatch between anatase and bronze phases where the mismatch was lowered with larger anatase crystals, thus enabling increased anatase capacity. The lithiation of polymer SDA-derived $\mathrm{TiO}_{2}$ has been examined many times, where the emergence of highly tailored sample series from PMT can reveal new opportunities and insights.

Such tunable isomorphic architectures are also an ideal platform for identifying rate-limiting steps in electrochemical devices without resorting to complex electrochemical modeling and the usual assumptions therein. For example, orthorhombic $\mathrm{T}-\mathrm{Nb}_{2} \mathrm{O}_{5}$ was the first material described as exhibiting intercalation pseudocapacitance, a faradaic charging process that is based upon intercalation yet often exhibits surface-limited kinetics [94]. Intercalation pseudocapacitance thus combines the high energy density a faradaic process with the high power density typical of capacitors. Curiously, materials such as $\mathrm{T}-\mathrm{Nb}_{2} \mathrm{O}_{5}$ exhibit pseudocapacitive responses over a wide range of conditions despite the fundamental inclusion of intercalation via diffusion. There have been numerous publications examining the electrochemical behavior of $\mathrm{T}-\mathrm{Nb}_{2} \mathrm{O}_{5}$ nanostructures produced using diverse methods, however, the general inclusion of multiple spatial variables or the lack of multiple sample conditions all together hamper 
the interpretation of nanostructure-dependent observations. Correspondingly, the claims for $\mathrm{T}-\mathrm{Nb}_{2} \mathrm{O}_{5}$ lithiation kinetics spanned from being intrinsically pseudocapacitive [95] and not requiring nanostructures for high rate capability [96] to being sluggish and requiring $24 \mathrm{~h}$ for lithiation [97], highlighting the need for a systematic study of nanostructuredependent behavior. The different steps of electrochemical processes are often investigated based upon their rate dependence where surface-limited steps and diffusion-limited steps each have a distinct time dependence [98]. For example, with cyclic voltammetry (CV) surface-limited processes have a current response (I) that is proportional to the sweep rate $(v)$ where I $\alpha v$ and semi-infinite diffusion-limited processes have a current response that is proportional to the square root of sweep rate where I $\alpha v^{0.5}$. Herein, lies the challenge: electrochemical devices typically have multiple diffusive processes with the same fundamental time dependence. Figure 3 shows the processes for lithiation of $\mathrm{T}-\mathrm{Nb}_{2} \mathrm{O}_{5}$ where there is one surface process and three diffusive processes that occur concomitantly. The diffusion processes include intercalation of lithium into the walls, diffusion of lithium ions through the electrolyte in the pores, and electron transport in the walls through the films thickness. Typical electrochemical analytical methods can identify transitions between surface-limited and diffusion-limited behavior but cannot alone identify the onset of a specific underlying diffusive process. For example, electrochemical impedance spectroscopy (EIS) data are often fitted with equivalent circuits where a significant challenge is that many datasets are well fitted by numerous equivalent circuits and there is often ambiguity as to which circuit element corresponds to which physical process [56]. EIS data interpreted in a less model-dependent fashion using 3D Bode plots also have the same challenge. A recent 3D Bode analysis of $\mathrm{T}-\mathrm{Nb}_{2} \mathrm{O}_{5}$ concluded with ambiguity that the sample rate capability was either limited by the intercalation diffusion or the electrolyte diffusion [57]. These examples highlight the challenges in assessing nanostructure-dependent performance of $\mathrm{T}-\mathrm{Nb}_{2} \mathrm{O}_{5}$ based on electrochemical modeling alone.

A set of tunable isomorphic architectures were prepared with PMTs to identify the first $\mathrm{T}-\mathrm{Nb}_{2} \mathrm{O}_{5}$ nanostructuredependent performance [55]. A set of samples with varied wall thickness were analyzed using $\mathrm{CV}$. The voltage sweeprate dependence of the anodic peak current response was analyzed to identify the power-law dependence (I $\alpha v^{\mathrm{b}}$ ) where $b=1.0$ for a surface-limited response and $b=0.5$ for a diffusion-limited response. The typical sample behavior
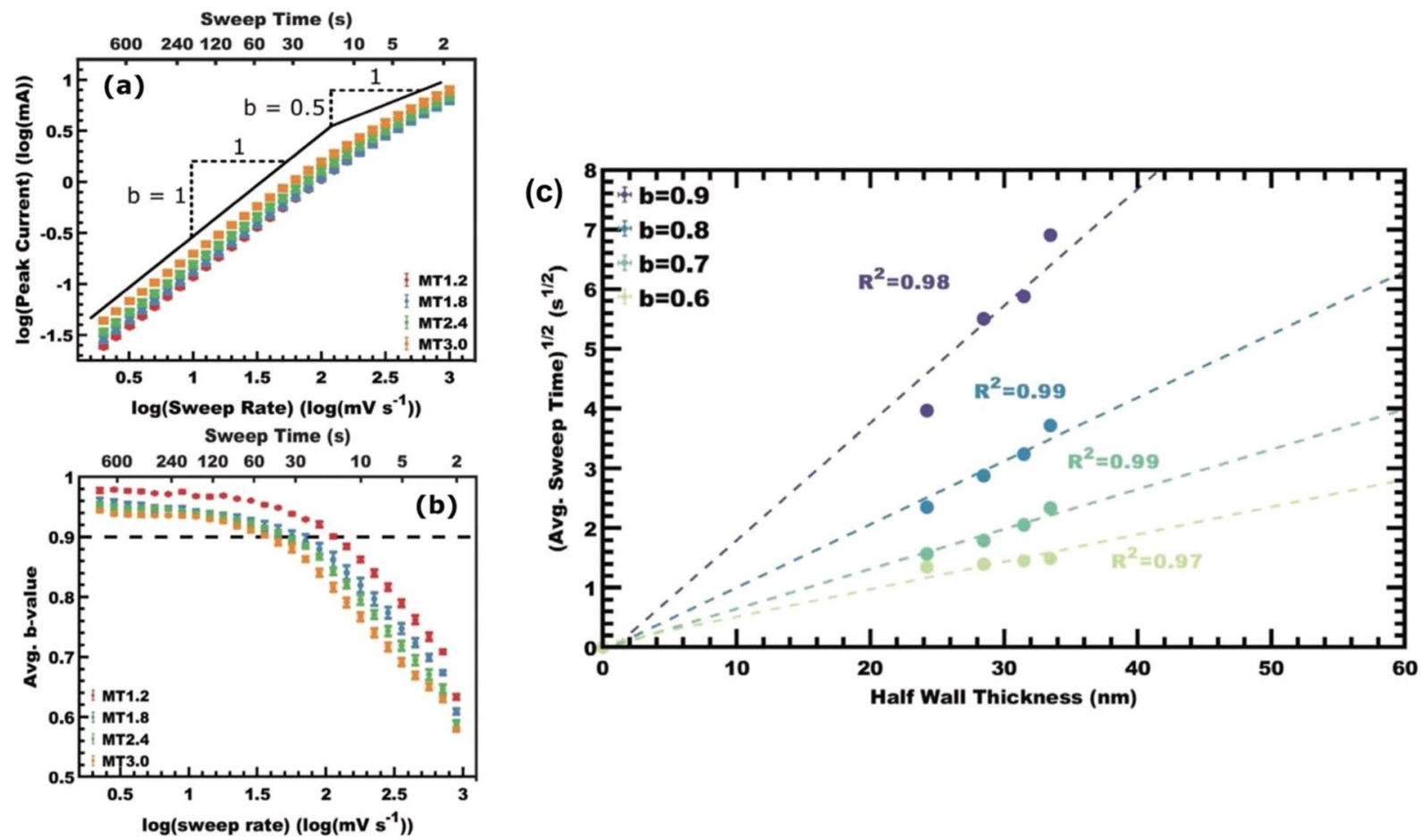

Figure 13: The electrochemical kinetics of a series of $\mathrm{T}-\mathrm{Nb}_{2} \mathrm{O}_{5}$ samples prepared using PEO- $b$-PHA PMTs were measured (a). The derivative of (a) corresponds to the $b$-value (b) where $b$-values proximal to 1.0 are consistent with surface-limited kinetics and values proximal to 0.5 are consistent with semi-infinite diffusion-limited kinetics. The surface-limited threshold (SLT, $b=0.9$ ) and diffusion-limited threshold (DLT, $b=0.6)$ were sensitively dependent upon the nanoscale architecture. Control experiments identified solid-state lithium diffusion as the dominant diffusion rate-limiting step. Both the DLT and the SLT performance metrics correlated to the diffusion length (half wall thickness) and were well fitted by Fick's second law (c). Such maps correlating nanostructure to performance (c) support the development of nano-optimized devices. Adapted from van den Bergh et al. from Ref. [55] with permission from John Wiley and Sons. 
was a pseudocapacitive surface-limited response at moderate voltage sweep rates and then transitioned towards diffusionlimited behavior at the fastest voltage sweep rates (Fig. 13a, b). The voltage sweep rate at which the samples departed from surface-limited behavior varied monotonically with the M:T ratio. This surface-limited threshold (SLT) thus represents that maximum voltage sweep rate $\left(v_{\text {SLT }}\right)$ at which a sample exhibits a surface-limited response, defined at $b=0.9$. Here, there was a remarkable $3 \times$ difference in $v_{\mathrm{SLT}}$ for the different PMT architectures despite the minor $18.5 \mathrm{~nm}$ change in wall thickness. Subsequent experiments were used to probe the effects of electrolyte concentration and film thickness to use the process of elimination to identify solid-state lithium diffusion as the dominant diffusion constraint, even for samples with just 48.5-67.0-nm-thick walls. With the specific diffusion process identified, the architectural dimensions were correlated to the observed behaviors. Fick's second law for 1D diffusion with an infinite source predicts that the diffusion length (x) relates to the diffusion time ( $\mathrm{t}$ ) and the diffusivity (D) where $x a \sqrt{ }(D t)$. Thus, a plot comparing the square root of sweep time (voltage window divided by voltage sweep rate) versus the diffusion length (half of the wall thickness) is expected to yield a relationship of direct proportionality (Fig. 13c). Here, the diffusion-limited threshold (DLT) is the minimum voltage sweep rate at which the behavior is diffusion-limited and is defined at $b=0.6$. As expected for a diffusive process, the data exhibited a direct proportionality relationship on these axes with an excellent goodness-of-fit $R^{2}$ of 0.97 . Interestingly, the SLT sweep time was also well modeled by this relationship $\left(R^{2}=0.98\right)$ suggesting that the maximum rate for surfacelimited behavior is determined by the onset of a solid-state diffusion constraint. Furthermore, Fig. 13c is also a tool for the production of nano-optimized architectures for target response times. This was the first publication showing how a series of tailored $\mathrm{T}-\mathrm{Nb}_{2} \mathrm{O}_{5}$ architectures result in widely varying intercalation pseudocapacitance kinetics. For interested readers, it is noted that niobium oxides have diverse stoichiometries and polymorphs with wide-ranging conductivity [99] and that the $\mathrm{T}-\mathrm{Nb}_{2} \mathrm{O}_{5}$ surface can change with electrochemical cycling [100]. Here, the use of a single synthesis technique is ideal to support comparisons between nearly identical samples. This example shows how alteration of a single nanostructure spatial variable at a time enables a refreshed perspective on electrochemical performance with minimal assumptions and without implementing complex electrochemical models.

\section{Conclusions}

A range of behaviors were reviewed for block polymer SDAs when combined with material precursors. These behaviors span the gamut from bulk-type coassembly and dynamic micelle coassembly to persistent micelle templates, in the order of increasing solvent selectivity. These distinct behaviors are associated with both the thermodynamic equilibrium as well as the kinetic rates of several processes. Recent developments with persistent micelle templates were highlighted where independent control over pore size and wall thickness were robustly developed in recent years. The validation of consistency with PMT behavior by diffraction models was described. The low barrier to entry for PMT processing was noted where both polymer SDA synthesis and SDA usage can be carried out using commodity supplies in less than $24 \mathrm{~h}$. It is notable that PMT procedures are simple and low cost with potential for future industrial upscaling. Future promising directions for PMT development include a broader range of feature sizes, additional morphologies, and control over multiple materials. Persistent micelles themselves have future applications in drug delivery or as nanoscale reactors. The tunable isomorphic architectures resulting from PMTs are enabling improved studies of nanostructure-dependent properties where examples were given for confinement effects during lithiation of $\mathrm{TiO}_{2}$ and the length-scale-dependent intercalation pseudocapacitance of $\mathrm{T}-\mathrm{Nb}_{2} \mathrm{O}_{5}$. Future promising applications for such tunable isomorphic architectures include the nanostructure-dependent study of catalysts, hydrogen storage, and drug delivery via porous particles. The ability to tailor architectures with one spatial variable at a time brings a unique perspective to inquiries and can provide unambiguous interpretations of nanoscale cause-and-effect.

\section{Acknowledgments}

M.S. acknowledges support by the NSF CAREER program, NSF Award No. DMR-1752615.

\section{Declarations}

Conflict of interest The corresponding author states that there is no conflict of interest.

\section{Open Access}

This article is licensed under a Creative Commons Attribution 4.0 International License, which permits use, sharing, adaptation, distribution and reproduction in any medium or format, as long as you give appropriate credit to the original author(s) and the source, provide a link to the Creative Commons licence, and indicate if changes were made. The images or other third party material in this article are included in the article's Creative Commons licence, unless indicated otherwise in a credit line to the material. If material is not included in the article's Creative Commons licence and your intended use is not permitted by statutory regulation or exceeds the permitted use, you will 
need to obtain permission directly from the copyright holder. To view a copy of this licence, visit http://creativecommons.org/ licenses/by/4.0/.

\section{References}

1. L.A. Kolahalam, I.V.K. Viswanath, B.S. Diwakar, B. Govindh, V. Reddy, Y.L.N. Murthy, Mater. Today: Proc. (2019). https://doi. org/10.1016/j.matpr.2019.07.371

2. N. Baig, I. Kammakakam, W. Falath, Mater. Adv. (2021). https:// doi.org/10.1039/D0MA00807A

3. J.A. Darr, J. Zhang, N.M. Makwana, X. Weng, Chem. Rev. (2017). https://doi.org/10.1021/acs.chemrev.6b00417

4. C.D. De Souza, B.R. Nogueira, M.E. Rostelato, J. Alloys Compd. (2019). https://doi.org/10.1016/j.jallcom.2019.05.153

5. I. Cesar, K. Sivula, A. Kay, R. Zboril, M. Grätzel, J. Phys. Chem. C (2009). https://doi.org/10.1021/jp809060p

6. W.-J. An, E. Thimsen, P. Biswas, J. Phys. Chem. Lett. (2010). https://doi.org/10.1021/jz900156d

7. H. Zhang, H. Zhou, Y. Wang, S. Li, P. Biswas, Energy Fuels (2020). https://doi.org/10.1021/acs.energyfuels.0c03264

8. L. Zhang, L. Jin, B. Liu, J. He, Front. Chem. (2019). https://doi. org/10.3389/fchem.2019.00022

9. X. Zhou, N.T. Nguyen, S. Özkan, P. Schmuki, Electrochemistry (2014). https://doi.org/10.1016/j.elecom.2014.06.021

10. D. Ongari, L. Talirz, B. Smit, ACS Cent. Sci. (2020). https://doi. org/10.1021/acscentsci.0c00988

11. T. Ghanbari, F. Abnisa, W.M.A.W. Daud, Sci. Total Environ. (2020). https://doi.org/10.1016/j.scitotenv.2019.135090

12. H.N. Lokupitiya, A. Jones, B. Reid, S. Guldin, M. Stefik, Chem. Mater. 28(6), 1653-1667 (2016). https://doi.org/10.1021/acs. chemmater.5b04407

13. F.S. Bates, G.H. Fredrickson, Phys. Today (1999). https://doi. org/10.1063/1.882522

14. M. Templin, A. Franck, A. Du Chesne, H. Leist, Y. Zhang, R. Ulrich, V. Schadler, U. Wiesner, Science (1997). https://doi.org/ 10.1126/science.278.5344.1795

15. D. Zhao, J. Feng, Q. Huo, N. Melosh, G.H. Fredrickson, B.F. Chmelka, G.D. Stucky, Science (1998). https://doi.org/10.1126/ science.279.5350.548

16. T. Yanagisawa, T. Shimizu, K. Kuroda, C. Kato, Bull. Chem. Soc. Jpn. (1990). https://doi.org/10.1246/bcsj.63.988

17. J.S. Beck, J.C. Vartuli, W.J. Roth, M.E. Leonowicz, C.T. Kresge, K.D. Schmitt, C.T.W. Chu, D.H. Olson, E.W. Sheppard, J. Am. Chem. Soc. (1992). https://doi.org/10.1021/ja00053a020

18. C.T. Kresge, M.E. Leonowicz, W.J. Roth, J.C. Vartuli, J.S. Beck, Nature (London) (1992). https://doi.org/10.1038/359710a0

19. S.A. Bagshaw, E. Prouzet, T.J. Pinnavaia, Science (1995). https:// doi.org/10.1126/science.269.5228.1242

20. G.D. Stucky, P. Yang, D. Zhao, D.I. Margolese, B.F. Chmelka, Nature (London) (1998). https://doi.org/10.1038/24132
21. G.J.D.A.A. Soler-Illia, C. Sanchez, New J. Chem. (2000). https:// doi.org/10.1039/b002518f

22. B. Smarsly, D. Grosso, T. Brezesinski, N. Pinna, Chem. Mater. (2004). https://doi.org/10.1021/cm0495966

23. C. Reitz, J. Haetge, C. Suchomski, T. Brezesinski, Chem. Mater. (2013). https://doi.org/10.1021/cm402995a

24. T. Brezesinski, J. Wang, J. Polleux, B. Dunn, S.H. Tolbert, J. Am. Chem. Soc. (2009). https://doi.org/10.1021/ja8057309

25. M. Dai, L. Song, J.T. LaBelle, B.D. Vogt, Chem. Mater. (2011). https://doi.org/10.1021/cm2002429

26. S. Choi, M. Mamak, N. Coombs, N. Chopra, G. Ozin, Adv. Funct. Mater. (2004). https://doi.org/10.1002/(ISSN)1616-3028

27. L. Li, M. Krissanasaeranee, S.W. Pattinson, M. Stefik, U. Wiesner, U. Steiner, D. Eder, Chem. Commun. (Camb) (2010). https://doi.org/10.1039/c0cc01237h

28. J.M. Szeifert, D. Fattakhova-Rohlfing, D. Georgiadou, V. Kalousek, J. Rathouský, D. Kuang, S. Wenger, S.M. Zakeeruddin, M. Grätzel, T. Bein, Chem. Mater. (2009). https://doi.org/ $10.1021 / \mathrm{cm} 8029246$

29. E. Lim, H. Kim, C. Jo, J. Chun, K. Ku, S. Kim, H.I. Lee, I.S. Nam, S. Yoon, K. Kang, J. Lee, ACS Nano (2014). https://doi.org/10. 1021/nn501972w

30. K. Peters, H.N. Lokupitiya, D. Sarauli, M. Labs, M. Pribil, J. Rathouský, A. Kuhn, D. Leister, M. Stefik, D. Fattakhova-Rohlfing, Adv. Funct. Mater. (2016). https://doi.org/10.1002/adfm. 201602148

31. Y. Deng, C. Liu, D. Gu, T. Yu, B. Tu, D. Zhao, J. Mater. Chem. (2008). https://doi.org/10.1039/B713310C

32. C. Liang, K. Hong, G.A. Guiochon, J.W. Mays, S. Dai, Angew. Chem. Int. Ed. Engl. (2004). https://doi.org/10.1002/anie.20046 1051

33. S.C. Warren, L.C. Messina, L.S. Slaughter, M. Kamperman, Q. Zhou, S.M. Gruner, F.J. DiSalvo, U. Wiesner, Science (2008). https://doi.org/10.1126/science.1159950

34. Y. Lin, V.K. Daga, E.R. Anderson, S.P. Gido, J.J. Watkins, J. Am. Chem. Soc. (2011). https://doi.org/10.1021/ja2003632

35. T. Brezesinski, D. Fattakhova Rohlfing, S. Sallard, M. Antonietti, B.M. Smarsly, Small (2006). https://doi.org/10.1002/smll.20060 0176

36. K. Brezesinski, J. Wang, J. Haetge, C. Reitz, S.O. Steinmueller, S.H. Tolbert, B.M. Smarsly, B. Dunn, T. Brezesinski, J. Am. Chem. Soc. (2010). https://doi.org/10.1021/ja9106385

37. C. Reitz, P.M. Leufke, H. Hahn, T. Brezesinski, Chem. Mater. (2014). https://doi.org/10.1021/cm500381g

38. K. Letchford, H. Burt, Eur. J. Pharm. Biopharm. (2007). https:// doi.org/10.1016/j.ejpb.2006.11.009

39. K. Zhang, X. Tang, J. Zhang, W. Lu, X. Lin, Y. Zhang, B. Tian, H. Yang, H. He, J. Controll. Release 183, 77-86 (2014)

40. Y. Xu, C. Kim, D.M. Saylor, D. Koo, J. Biomed. Mater. Res. B 105, 1692-1716 (2017) 
41. D.A. Olson, L. Chen, M.A. Hillmyer, Chem. Mater. (2008). https://doi.org/10.1021/cm702239k

42. E.A. Jackson, M.A. Hillmyer, ACS Nano (2010). https://doi.org/ 10.1021/nn1014006

43. E.J.W. Crossland, S. Ludwigs, M.A. Hillmyer, U. Steiner, Soft Matter (2007). https://doi.org/10.1039/b609780d

44. A. Sarkar, M. Stefik, Mater. Chem. Front. (2017). https://doi. org/10.1039/C6QM00266H

45. F.S. Bates, MRS Bull. (2005). https://doi.org/10.1557/mrs2005. 145

46. P.C. Hiemenz, T.P. Lodge, Polymer Chemistry (CRC Press, New York, 2007)

47. P.C.A. Alberius, K.L. Frindell, R.C. Hayward, E.J. Kramer, G.D. Stucky, B.F. Chmelka, Chem. Mater. (2002). https://doi.org/10. 1021/cm011209u

48. B.C. Garcia, M. Kamperman, R. Ulrich, A. Jain, S.M. Gruner, U. Wiesner, Chem. Mater. (2009). https://doi.org/10.1021/cm901 $885 \mathrm{c}$

49. M. Stefik, S. Mahajan, H. Sai, T.H. Epps, F.S. Bates, S.M. Gruner, F.J. Disalvo, U. Wiesner, Chem. Mater. (2009). https://doi.org/ $10.1021 / \mathrm{cm} 902626 \mathrm{z}$

50. R.C. Hayward, D.J. Pochan, Macromolecules (2010). https://doi. org/10.1021/ma9026806

51. D. Grosso, G.J.D.A.A. Soler-Illia, E.L. Crepaldi, F. Cagnol, C. Sinturel, A. Bourgeois, A. Brunet-Bruneau, H. Amenitsch, P.A. Albouy, C. Sanchez, Chem. Mater. (2003). https://doi.org/10. $1021 / \mathrm{cm} 031060 \mathrm{~h}$

52. M. Stefik, J. Song, H. Sai, S. Guldin, P. Boldrighini, M.C. Orilall, U. Steiner, S.M. Gruner, U. Wiesner, J. Mater. Chem. A (2015). https://doi.org/10.1039/C5TA02483H

53. S.W. Boettcher, J. Fan, C.K. Tsung, Q. Shi, G.D. Stucky, Acc. Chem. Res. (2007). https://doi.org/10.1021/ar6000389

54. S. Guldin, P. Docampo, M. Stefik, G. Kamita, U. Wiesner, H.J. Snaith, U. Steiner, Small (2012). https://doi.org/10.1002/smll. 201102063

55. W. van den Bergh, H.N. Lokupitiya, N.A. Vest, B. Reid, S. Guldin, M. Stefik, Adv. Funct. Mater. (2021). https://doi.org/10. 1002/adfm.202007826

56. B.-A. Mei, J. Lau, T. Lin, S.H. Tolbert, B.S. Dunn, L. Pilon, J. Phys. Chem. C (2018). https://doi.org/10.1021/acs.jpcc.8b05241

57. J.S. Ko, C.-H. Lai, J.W. Long, D.R. Rolison, B. Dunn, J. Nelson Weker, ACS Appl. Mater. Interfaces (2020). https://doi.org/10. 1021/acsami.0c02020

58. De Yoreo J, Mandrus D, Soderholm L, Forbes T, Kanatzidis M, Erlebacher J, Laskin J, Wiesner U, Xu T, Billinge S (2016) Basic research needs workshop on synthesis science for energy relevant technology. USDOE Office of Science

59. B.S. Guiton, M. Stefik, V. Augustyn, S. Banerjee, C.J. Bardeen, B.M. Bartlett, J. Li, V. López-Mejías, L.R. MacGillivray, A. Morris, MRS Bull. (2020). https://doi.org/10.1557/mrs.2020.271
60. A. Vu, Y. Qian, A. Stein, Adv. Energy Mater. (2012). https://doi. org/10.1002/aenm.201200320

61. E.J.W. Crossland, M. Nedelcu, C. Ducati, S. Ludwigs, M.A. Hillmyer, U. Steiner, H.J. Snaith, Nano Lett. (2009). https://doi. org/10.1021/nl800942c

62. O. Kim, S.Y. Kim, J. Lee, M.J. Park, Chem. Mater. (2016). https://doi.org/10.1021/acs.chemmater.5b04157

63. A. Sarkar, M. Stefik, J. Mater. Chem. A (2017). https://doi.org/ 10.1039/C7TA01034F

64. Stefik M, Lokupitiya HN (2021) US Patent 10,954,393

65. J. Zhang, Y. Deng, J. Wei, Z. Sun, D. Gu, H. Bongard, C. Liu, H. Wu, B. Tu, F. Schuth, D. Zhao, Chem. Mater. (2009). https://doi. org $/ 10.1021 / \mathrm{cm} 901371 \mathrm{r}$

66. R. Buonsanti, T.E. Pick, N. Krins, T.J. Richardson, B.A. Helms, D.J. Milliron, Nano Lett. (2012). https://doi.org/10.1021/nl302 206s

67. G.K. Ong, T.E. Williams, A. Singh, E. Schaible, B.A. Helms, D.J. Milliron, Nano Lett. (2015). https://doi.org/10.1021/acs.nanol ett.5b03765

68. E. Ortel, A. Fischer, L. Chuenchom, J. Polte, F. Emmerling, B. Smarsly, R. Kraehnert, Small (2012). https://doi.org/10.1002/ smll.201101520

69. D. Bernsmeier, E. Ortel, J. Polte, B. Eckhardt, S. Nowag, R. Haag, R. Kraehnert, J. Mater. Chem. A (2014). https://doi.org/ 10.1039/C4TA01842G

70. S.-H. Choi, T.P. Lodge, F.S. Bates, Phys. Rev. Lett. (2010). https://doi.org/10.1103/PhysRevLett.104.047802

71. J. Lu, F.S. Bates, T.P. Lodge, ACS Macro Lett. (2013). https://doi. org/10.1021/mz400167x

72. Y. Ma, T.P. Lodge, Macromolecules (2016). https://doi.org/10. 1021/acs.macromol.6b02212

73. A. Halperin, S. Alexander, Macromolecules (1989). https://doi. org/10.1021/ma00195a069

74. T. Haliloglu, I. Bahar, B. Erman, W.L. Mattice, Macromolecules (1996). https://doi.org/10.1021/ma951301

75. E.E. Dormidontova, Macromolecules (1999). https://doi.org/10. 1021/ma9809029

76. A.G. Denkova, E. Mendes, M.-O. Coppens, Soft Matter. (2010). https://doi.org/10.1039/c001175b

77. C.J. Brinker, G.W. Scherer, Sol-Gel Science (Academic Press, New York, 2013)

78. J. Brandrup, E.H. Immergut, E.A. Grulke, A. Abe, D.R. Bloch, Polymer Handbook (Wiley, New York, 1999)

79. S. Guldin, S. Hüttner, P. Tiwana, M.C. Orilall, B. Ülgüt, M. Stefik, P. Docampo, M. Kolle, G. Divitini, C. Ducati, S.A.T. Redfern, H.J. Snaith, U. Wiesner, D. Eder, U. Steiner, Energy Environ. Sci. (2010). https://doi.org/10.1039/c0ee00362j

80. S. Guldin, M. Stefik, H. Sai, U. Wiesner, U. Steiner, RSC Adv. (2015). https://doi.org/10.1039/C5RA00836K 
81. A. Sarkar, L. Evans, M. Stefik, Langmuir 34(20), 5738-5749 (2018). https://doi.org/10.1021/acs.langmuir.8b00417

82. A.F.M. Barton, CRC Handbook of Solubility Parameters and Other Cohesion Parameters (Routledge, New York, 2017)

83. K.A. Lantz, N.B. Clamp, W. van den Bergh, A. Sarkar, M. Stefik, Small (2019). https://doi.org/10.1002/smll.201900393

84. A. Sarkar, A. Thyagarajan, A. Cole, M. Stefik, Soft Matter (2019). https://doi.org/10.1039/c9sm00484j

85. Y. Deng, J. Liu, C. Liu, D. Gu, Z. Sun, J. Wei, J. Zhang, L. Zhang, B. Tu, D. Zhao, Chem. Mater. (2008). https://doi.org/10.1021/ $\mathrm{cm} 802413 \mathrm{q}$

86. J. Wei, Y. Deng, J. Zhang, Z. Sun, B. Tu, D. Zhao, Solid State Sci. (2011). https://doi.org/10.1016/j.solidstatesciences.2010.03.008

87. L.B. McCusker, F. Liebau, G. Engelhardt, Pure Appl. Chem. (2001). https://doi.org/10.1016/S1387-1811(02)00545-0

88. H.N. Lokupitiya, M. Stefik, Nanoscale (2017). https://doi.org/ 10.1039/c6nr07313a

89. K.A. Lantz, A. Sarkar, K.C. Littrell, T. Li, K. Hong, M. Stefik, Macromolecules (2018). https://doi.org/10.1021/acs.macromol. 8 b01244

90. E.R. Williams, P.L. McMahon, J. Reynolds III., J.L. Snider, V. Stavila, M.D. Allendorf, M. Stefik, Mater. Adv. (2021). https:// doi.org/10.1039/D1MA00146A

91. T. Larison, M. Stefik, Langmuir (2021). https://doi.org/10.1021/ acs.langmuir.1c01384
92. R.P. Murphy, E.G. Kelley, S.A. Rogers, M.O. Sullivan, T.H. Epps, ACS Macro Lett. (2014). https://doi.org/10.1021/mz500435d

93. Stefik M. (2021). http://www.stefikgroup.com/spin-coater/. Accessed 9 Nov 2021

94. V. Augustyn, J. Come, M.A. Lowe, J.W. Kim, P.-L. Taberna, S.H. Tolbert, H.D. Abruna, P. Simon, B. Dunn, Nat. Mater. (2013). https://doi.org/10.1038/nmat3601

95. J. Come, V. Augustyn, J.W. Kim, P. Rozier, P.-L. Taberna, P. Gogotsi, J.W. Long, B. Dunn, P. Simon, J. Electrochem. Soc. (2014). https://doi.org/10.1149/2.040405jes

96. K.J. Griffith, A.C. Forse, J.M. Griffin, C.P. Grey, J. Am. Chem. Soc. (2016). https://doi.org/10.1021/jacs.6b04345

97. B. Reichman, A.J. Bard, J. Electrochem. Soc. (1981). https://doi. org/10.1149/1.2127416

98. A.J. Bard, L.R. Faulkner, Electrochemical Methods: Fundamentals and Applications, 2nd edn. (Wiley Global Education, New York, 2000)

99. C. Nico, T. Monteiro, M.P.F. Graça, Prog. Mater. Sci. (2016). https://doi.org/10.1016/j.pmatsci.2016.02.001

100. I. Andoni, J.M. Ziegler, G. Jha, C.A. Gadre, H. Flores-Zuleta, S. Dai, S. Qiao, M. Xu, V.T. Chen, X. Pan, R.M. Penner, ACS Appl Energy Mater (2021). https://doi.org/10.1021/acsaem.1c00580 